\title{
Nominal Performance of Future Dual Frequency Dual Constellation GBAS
}

\author{
Daniel Gerbeth, Mihaela-Simona Circiu, Maria Caamano, and Michael Felux
}

German Aerospace Center (DLR), Münchner Straße 20, 82234 Weßling, Germany

Correspondence should be addressed to Daniel Gerbeth; daniel.gerbeth@dlr.de

Received 1 April 2016; Accepted 15 May 2016

Academic Editor: Yanbo Zhu

Copyright (C) 2016 Daniel Gerbeth et al. This is an open access article distributed under the Creative Commons Attribution License, which permits unrestricted use, distribution, and reproduction in any medium, provided the original work is properly cited.

In this work an overview of numerous possible processing modes in future dual frequency, dual constellation GBAS is given and compared to the current GAST D standard. We discuss the individual error contributions to GBAS protection levels and give an overview of the general processing. Based on this the consequences when adding a second constellation as well as frequency are investigated. Geometrical implications and changes to the residual differential error bounds are studied separately first. In terms of geometry a comparison between the single and dual constellation case is presented using dilution of precision as metric. The influence on the different sigma contributions when using new satellites (Galileo) and signals (E1, L5, and E5a) is individually discussed based on recent measurements. Final simulations for different varying parameters are carried out to compare relevant processing modes in terms of achieved nominal protection levels. A concluding discussion compares the outcomes and analyzes the implications of choosing one or the other mode.

\section{Introduction}

The Ground Based Augmentation System (GBAS) is a navigation system for aircraft designed to be used for precision approaches and landings. At its core is a differential Global Navigation Satellite System (GNSS) architecture providing locally relevant corrections for the navigation signals from the satellites. Furthermore, the ground station monitors the signals for any condition which might be able to cause differential navigation errors. Based on the monitoring and a performance evaluation of the ground station, integrity parameters are transmitted along with the corrections which enable the airborne system to calculate bounds on the differential positioning error. This instantaneous error bounding allows the airborne system to determine if the safety requirements for the operation are met at any point in time.

Currently, GBAS ground stations supporting CAT-I operations (i.e., approaches down to a minimum decision height of not less than $200 \mathrm{ft}$ and a runway visual range (RVR) of at least $800 \mathrm{~m}$ ) are commercially available and in operation at several airports throughout Europe, the United States, Australia, and Russia (for the latest up-to-date information on operational stations see the website http://www.flygls.net/). In the GBAS terminology these stations support the GBAS
Approach Service Type (GAST) C. Development and standardization of a GBAS able to support CAT-II/III operations (i.e., operations with lower minima or without any requirements concerning visibility at all) are in their final stage. In GBAS terminology this service is called GAST D. It is expected that the standardization will be completed by the International Civil Aviation Organization (ICAO) by the end of 2016. Most of the current systems, however, only support the use of signals from the US-American Global Positioning System (GPS) on one frequency (L1). Under certain conditions this can lead to a somewhat reduced availability of the service, mainly caused by ionospheric irregularities in certain regions of the world.

All countries where a GBAS is in operation need to determine their own ionospheric threat space; that is, the largest gradient which can occur needs to be defined and the integrity parameters need to be adjusted accordingly. Several countries in mid-latitudes have performed this task [1-3]. Of all threat models in those regions the largest gradients were found in the United States. Applying this threat model to the station yields satisfactory performance for operational GAST C stations. However, recent studies investigated the ionospheric threat space in Brazil as an example for an equatorial region with very high ionospheric activity. In those 
studies gradients almost twice as large as the worst case in the US were found [4]. Furthermore, ionospheric scintillations cause frequent loss of lock events in GNSS receivers. These effects occur very regularly after sunset and impact the availability of the service [5].

With the latest generation of GPS satellites (the socalled Block IIF) a second civil signal on a frequency usable for aviation has become available to users. Furthermore, new constellations such as the European Galileo system are currently being launched which provide interoperability and civil signals on two frequencies from the beginning. As the ionospheric delay is frequency dependent, it can be estimated and removed when using a dual frequency combination of the signals. In addition to that, the large number of satellites available for navigation will also provide significant benefits in terms of geometric diversity of the satellites and enable good and reliable positioning even in the presence of ionospheric scintillations. For that reason significant work is underway in order to develop and standardize a future GBAS service which supports the use of two frequencies and several constellations in order to mitigate the negative effects on current systems.

The use of multiple constellations and two frequencies could enable a very large number of different processing modes which all would have to be analyzed in detail with all potential fault modes identified and bounded in the integrity concept. Furthermore, the new architecture has to be backwards compatible in order to be interoperable with existing ground stations and airborne equipment which is already in use. In order to keep the validation, standardization, and certification efforts manageable the number of new modes will be kept to a minimum. It is therefore important at this stage to carefully analyze all implications a new GBAS mode brings along. In this study we investigate the nominal performance of different processing modes in order to contribute to this effort of selecting an optimal future architecture based on the benefits that can be expected. Thus we combine the results from our previous studies with new measurement data to give a comprehensive overview of the performance for trade-off studies regarding the selection of processing modes.

We will start with a short discussion about the different processing modes considered and the baseline integrity concept in the form of calculating error bounds, the so-called protection levels. Next, we investigate benefits of a second constellation in terms of geometric diversity in different scenarios and the effect on the protection levels. Aside from the geometry the expected residual differential error bounds play an important role. We revisit the individual contributors and discuss the effects observed and changes to the current models. Finally, we show a comparison of several different processing modes and their protection levels in exemplary scenarios before our concluding discussion.

\section{GBAS Processing}

The GBAS corrects for the combined effects of multiple sources of measurement errors that are highly correlated

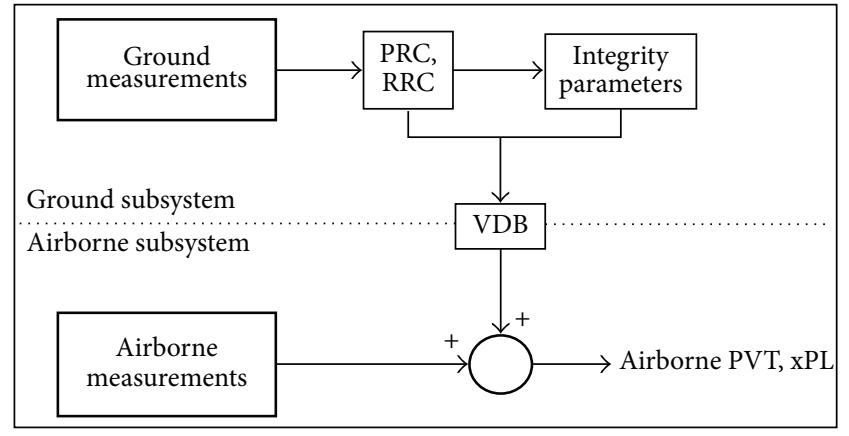

FIGURE 1: Simple overview diagram of GBAS architecture.

between reference receivers and users. These effects include satellite clock and ephemeris errors as well as ionospheric and tropospheric delay. Through precise knowledge about the location of the reference receivers at the airport it is possible to generate differential corrections for the GNSS measurements that are then broadcast to the airborne users. The onboard system applies the received corrections to its own measurements and thus corrects for the largest part of measurement errors and makes the position estimate very precise and reliable. A detailed description of the current processing modes is given, for example, in [6] and a schematic overview is given in Figure 1.

The current GBAS architecture is based on single frequency GPS L1 C/A code measurements only. Single frequency smoothing reduces noise and multipath; however ionospheric effects can potentially cause differential errors if the plasma density in the ionosphere exhibits large gradients. The use of a second frequency in GBAS processing might overcome many current limitations of the single frequency system. One of the dual frequency smoothing algorithms that have been proposed to mitigate the effect of the ionosphere gradients is the ionosphere-free (Ifree) combination. By forming a linear combination of the code and phase measurements from two frequencies the Ifree combination removes the ionospheric error (except for higher order effects that are negligible in this context).

In order to reduce noise and multipath errors, codecarrier smoothing is performed in the ground station and at the airborne receiver. Smoothing makes use of the less noisy but ambiguous carrier-phase measurements to suppress the noise and multipath from the noisy but unambiguous code measurements. In general, the input is the difference of the code and carrier-phase measurements, which is called "code minus carrier" or CMC. The final smoothed pseudoranges are obtained by adding the carrier-phase measurements back into the smoothed CMC. This step restores the range to the satellite and removes the integer ambiguity.

The expression of the smoothing filter as defined in DO$253 \mathrm{C}[7]$ is

$$
\widehat{\rho}_{n}=\frac{\Delta t}{\tau} \rho_{\mathrm{in}, n}+\left(1-\frac{\Delta t}{\tau}\right)\left[\hat{\rho}_{n-1}+\lambda\left(\phi_{\mathrm{in}, n}-\phi_{\mathrm{in}, n-1}\right)\right],
$$

where $\hat{\rho}_{n}$ is the current carrier-smoothed pseudorange (in meters), $\widehat{\rho}_{n-1}$ is the previous carrier-smoothed pseudorange 
(in $\mathrm{m}$ ), $\rho_{\mathrm{in}, n}$ is the current raw pseudorange measurement (in $\mathrm{m}$ ), $\phi_{\mathrm{in}, n}$ is the current phase measurement input (in cycles), $\phi_{\text {in } n-1}$ is the previous carrier-phase measurement (in cycles), $\lambda$ is the wavelength (in $\mathrm{m}$ ), $\Delta t$ represents the sample interval (in seconds), and $\tau$ is the filter time constant (100 seconds or 30 seconds). We will now have a look at the errors contained in the final smoothed pseudoranges for the different smoothing techniques (single frequency and dual frequency).

2.1. Single Frequency Smoothing. In single frequency smoothing, the code minus carrier method is performed using code and carrier-phase measurements from the same frequency. Since the ionosphere affects code and carrier by the same amount but with opposite sign, the filter input (CMC) will contain double the ionospheric delay as expressed in (2). In this equation, $I_{f_{i}}$ represents the ionospheric delay, $N_{f_{i}}$ is the carrier-phase integer ambiguity, and $\mathrm{MP} \rho_{\rho_{f_{i}}}$ and $\epsilon_{\rho_{f_{i}}}$ are the code multipath and noise. Carrier-phase error (multipath and noise) is neglected and omitted in the equation, as it is small enough (typically in the range of millimeters) in the GBAS context to be ignored:

$$
\mathrm{CMC}_{f_{i}}=\rho_{f_{i}}-\phi_{f_{i}}=2 I_{f_{i}}-N_{f_{i}} \lambda_{f_{i}}+\mathrm{MP}_{\rho_{f_{i}}}+\epsilon_{\rho_{f_{j}}} .
$$

After recombining the ranging information by adding the carrier input to the smoothed CMC, the carrier-smoothed code measurements $\widehat{\rho}_{f_{i}}$ are expressed by (3). Here $R$ represents the geometric range from a user to the satellite, $T$ the tropospheric error, $\Delta t^{\mathrm{SV}}$ the satellite clock bias, $\Delta t_{\mathrm{rx}}$ the receiver clock bias, $\widehat{\mathrm{MP}}_{\rho_{f_{i}}}$ the smoothed code multipath, $\widehat{\epsilon}_{\rho_{f_{i}}}$ the smoothed noise on code, $I_{f_{i}}$ the (unknown) ionospheric error on the raw code measurement, and $\widehat{I}_{f_{i}}$ the ionospheric error on code after smoothing:

$$
\begin{aligned}
\widehat{\rho}_{f_{i}}= & R+T+c\left(\Delta t_{\mathrm{rx}}-\Delta t^{\mathrm{SV}}\right)+\widehat{\mathrm{MP}}_{\rho_{f_{i}}}+\widehat{\epsilon}_{\rho_{f_{i}}}+2 \widehat{I}_{f_{i}} \\
& -I_{f_{i}} .
\end{aligned}
$$

If the ionospheric error is constant over time, the lowpass filter would not have any impact on it and $\widehat{I}_{f_{i}}=I_{f_{i}}$ would hold. The observed ionospheric delay introduced on a measurement varies with time of the day and elevation of the satellite. Furthermore the single frequency filter introduces an additional delay due to the difference between its (averaged) impact on smoothed code and its impact on carrier. This effect is called code-carrier divergence.

2.2. Ionosphere Frequency Smoothing. Ionosphere-free smoothing completely removes the first-order effects of the ionospheric delays by using the code and carrier-phase ionosphere-free combinations from two frequencies as inputs to the smoothing filter. The code and carrier-phase inputs are described in (4), where $\rho_{f_{i}}$ and $\rho_{f_{j}}$ represent the code measurements on frequencies $f_{i}$ and $f_{j}$ and $\phi_{f_{i}}$ and $\phi_{f_{j}}$ the respective carrier-phase measurements:

$$
\begin{gathered}
\phi_{\text {Ifree }}=\frac{f_{i}^{2} \phi_{f_{i}}-f_{j}^{2} \phi_{f_{j}}}{f_{i}^{2}-f_{j}^{2}}, \\
\rho_{\text {Ifree }}=\frac{f_{i}^{2} \rho_{f_{i}}-f_{j}^{2} \rho_{f_{j}}}{f_{i}^{2}-f_{j}^{2}} .
\end{gathered}
$$

The Ifree filter input $\left(\mathrm{CMC}_{\text {Ifree }}\right)$ is not affected by the ionospheric error; thus the delay due to the smoothing effect on the ionosphere is removed from the filter output. The final carrier-smoothed code measurements $\left(\widehat{\rho}_{\text {Ifree }}\right)$ are described in (5), where $R$ is the geometric range from user to satellite as before, $T$ is the tropospheric error, $\Delta t^{\mathrm{SV}}$ is the satellite clock bias, $\Delta t_{\mathrm{rx}}$ is the receiver clock bias, $\widehat{\mathrm{MP}}_{\rho_{f_{i}}}, \widehat{\mathrm{MP}}_{\rho_{f_{j}}}$, $\widehat{\epsilon}_{\rho_{f_{i}}}$, and $\widehat{\epsilon}_{\rho_{f_{j}}}$ are the smoothed multipath and noise errors on frequencies $f_{i}$ and $f_{j}$, respectively, and IFB represents the interfrequency code bias caused by hardware differences between the two frequencies which appears when combining code measurements across frequencies [8]:

$$
\begin{aligned}
\widehat{\rho}_{\text {Ifree }}= & R+T+c\left(\Delta t_{\mathrm{rx}}-\Delta t^{\mathrm{SV}}\right) \\
& +\frac{f_{i}^{2}}{f_{i}^{2}-f_{j}^{2}}\left(\widehat{\mathrm{MP}}_{\rho_{f_{i}}}+\widehat{\epsilon}_{\rho_{f_{i}}}\right) \\
& -\frac{f_{j}^{2}}{f_{i}^{2}-f_{j}^{2}}\left(\widehat{\mathrm{MP}}_{\rho_{f_{j}}}+\widehat{\epsilon}_{\rho_{f_{j}}}\right)+\mathrm{IFB} .
\end{aligned}
$$

The Ifree solution no longer contains ionospheric delays (to a first-order) but instead contains the combination of multipath and noise effects from two code measurements. This increases the standard deviation of the differential pseudorange error and thus also of the position solution and protection levels as will be analyzed more in detail later in this paper.

However, due to the VDB capacity it is unlikely to have corrections for a second frequency for both smoothing time constants [9]. As there is no decision made which smoothing time constant should be used for the second frequency we analyze the performance of both possible solutions, 30second and 100-second smoothed corrections. Based on the available single frequency corrections (L1/E1 and L5/E5a) Ifree corrections can be formed on the airborne side. In order to remove the ionospheric delay the single frequency corrections have to be generated using the same smoothing time constant [10].

\section{Error Bounding in GBAS and Performance Metric}

The system availability is one of the most important performance parameters. The system is available whenever no monitor triggers and the protection levels (PLs) calculated by the user are smaller than the alert limits (ALs) for the 
current phase of flight. The ALs depend on the distance of the aircraft to the runway and represent the maximum tolerable navigation errors which do not endanger the safety of the operation. Protection levels are bounds to the residual position error after application of the differential corrections provided by the ground system which are only exceeded with a risk of $2 \cdot 10^{-7}$ [11]. Because the vertical alert limit (VAL) is much tighter ( $10 \mathrm{~m}$ close to the runway threshold) than the lateral alert limit and the vertical errors are typically larger than lateral errors, only the VPL will be used as a measure to assess the performance of different processing modes. We keep the general overbounding concept and stick with the established processing techniques and parameters.

According to RTCA DO-253C [7] the protection levels for the fault-free case are computed as

$$
\mathrm{VPL}_{\mathrm{Apr} \_\mathrm{H} 0}=k_{\mathrm{ffmd}} \cdot \sqrt{\sum_{i=1}^{N} s_{\mathrm{vert}, i}^{2} \cdot \sigma_{i}^{2}}+D_{V} \text {, }
$$

where $k_{\text {ffmd }}$ represents the fault-free missed detection multiplier, $s_{\text {vert }}$ the vertical projection factor in the along-track coordinate system (see (8)), and $\sigma_{i}^{2}$ the standard deviation of the uncertainty of the residual differential error. The variance $\sigma^{2}$ consists of different error contributions

$$
\begin{aligned}
\sigma_{i}^{2} & =\sigma_{\text {pr_gnd } \_x, i_{1}^{2}}^{2}+\sigma_{\text {tropo }, i}^{2}+\sigma_{\text {pr_air }, i_{i}}^{2}+\sigma_{\text {iono }, i}^{2}, \\
s_{\text {vert }, i} & =s_{3, i}+\tan \left(\theta_{\mathrm{GPA}}\right) \cdot s_{1, i}
\end{aligned}
$$

based on the weighted pseudoinverse $\mathbf{S}$ of the geometry matrix $\mathbf{G}$. The parameter $\theta_{\mathrm{GPA}}$ represents the glide path angle (typically $3^{\circ}$ ), to account for the fact that an uncertainty in along-track direction projects into the vertical domain.

The $\mathbf{S}$ matrix translates the measurements from pseudorange domain into the position domain and is defined as

$$
\mathbf{S}=\left(\mathbf{G}^{T} \mathbf{W G}\right)^{-1} \mathbf{G}^{T} \mathbf{W}
$$

where each row $\mathbf{G}_{i}$ of the geometry matrix $\mathbf{G}$ is defined as

$$
\left[\begin{array}{lll}
-\cos \left(\mathrm{El}_{i}\right) \cos \left(\mathrm{Az}_{i}\right)-\cos \left(\mathrm{El}_{i}\right) \sin \left(\mathrm{Az}_{i}\right)-\sin \left(\mathrm{El}_{i}\right) & 1 & 0
\end{array}\right]
$$

for GPS or

$$
\left[-\cos \left(\mathrm{El}_{i}\right) \cos \left(\mathrm{Az}_{i}\right)-\cos \left(\mathrm{El}_{i}\right) \sin \left(\mathrm{Az}_{i}\right)-\sin \left(\mathrm{El}_{i}\right) \quad 0 \quad 1\right]
$$

for Galileo.

The parameters $\mathrm{Az}_{i}$ and $\mathrm{El}_{i}$ are azimuth and elevation of the $i$ th satellite, respectively.

The inverse of the least squares weighting matrix $\mathbf{W}$ is defined as

$$
\mathbf{W}^{-1}=\left[\begin{array}{cccc}
\sigma_{1}^{2} & 0 & \cdots & 0 \\
0 & \sigma_{2}^{2} & \cdots & 0 \\
\vdots & \vdots & \ddots & \vdots \\
0 & 0 & \cdots & \sigma_{N}^{2}
\end{array}\right]
$$

The terms $D_{V}$ (and $D_{L}$ in the lateral case) are GAST D specific terms that represent the magnitude of the vertical and lateral difference between 30-second and 100-second smoothed position solutions. Note that for future processing modes some additional nominal errors might have to be bounded for in the protection levels. This could be true, for example, for interfrequency biases in the dual frequency case. However, for the moment we limit the study to the same contributors that were shown in (6). These are now discussed in detail in the following section.

\section{Contributors to Error Budget}

When looking at the protection level calculation (6) two main influencing factors can be separated: the influence of the satellite geometry on the one hand and the different noise contributions (residual differential error bounds) on the other hand. While the geometry is dominantly defining the $\mathbf{S}$ matrix (9) the noise also plays a role as weighting factor here. In the final protection level equation the summed noise terms occur again, being scaled by the weighted $\mathbf{s}$-factors.

4.1. Geometry Projection Factors. First we consider the influence of adding a second constellation (in this study Galileo) in terms of satellite geometry. Even if all the simulations in this paper focus on Galileo it will be mentioned that the general influence on the geometry stays comparable when considering GPS and GLONASS or BeiDou instead.

The evaluations in this section utilize dilution of precision (DOP) values to separate the geometric effects on the protection level performance. In general DOP values represent an additional, multiplicative uncertainty which comes from the geometric distribution of the satellites. Therefore, smaller DOP values represent a more favorable geometry. These DOP values can be calculated for different domains, like horizontal, vertical, or 3D positioning. The so-called DOP-matrix $\mathbf{H}$ can be computed as

$$
\mathbf{H}=\left(\mathbf{G}^{T} \mathbf{G}\right)^{-1}
$$

using the same geometry matrix as in (9). In case of two constellations and therefore two clock offsets which have to be estimated the resulting matrix is of shape $\mathbb{R}^{(5 \times 5)}$.

The diagonal elements of $\mathbf{H}$ represent the different DOPs as follows:

H

$$
=\left[\begin{array}{ccccc}
\mathrm{NDOP}^{2} & & \cdots & & \\
& \mathrm{EDOP}^{2} & & & \\
\vdots & & & & \vdots \\
& & \operatorname{VDOP}^{2} & & \\
& & & \mathrm{TDOP}_{\mathrm{GPS}}^{2} & \\
& & \cdots & & \mathrm{TDOP}_{\mathrm{Gal}}^{2}
\end{array}\right]
$$

GDOP which takes into account the time DOPs is built up of all diagonal elements.

As in GBAS the vertical domain is the most critical and at the same time vertical positioning is least precise due to the 


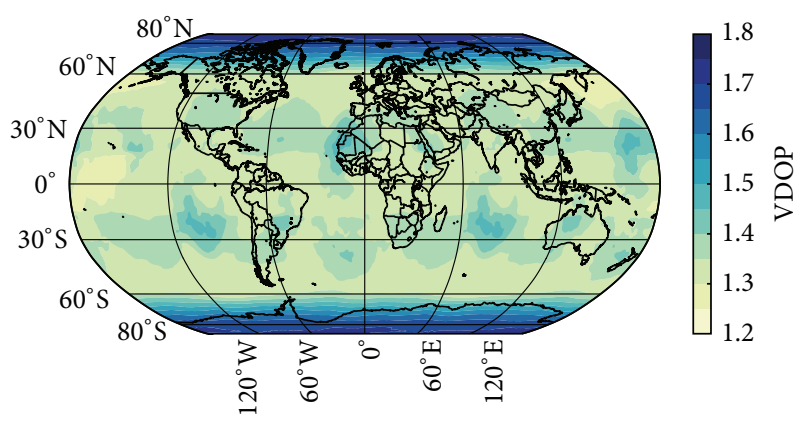

FIGURE 2: Global simulation of average VDOP using GPS only ( $5^{\circ}$ elevation mask).

satellite geometry (there are no satellites below us), further studies will focus on the vertical VDOP.

\subsubsection{Global DOP Values and Influence of Elevation Masking.} Figure 2 shows the global distribution of average VDOPs. The VDOP values in this plot are calculated on a 2-by-2degree grid using all satellites in view for a GPS constellation with 31 satellites. Time-wise averaging is performed over 10 days, taking one sample every 150 seconds with a typical elevation masking of $5^{\circ}$. As can be seen from the plot the VDOP fluctuates only slightly in latitudes between $60^{\circ}$ south and north in the range of 1.2 to 1.4 for most regions. Going closer to the poles the VDOP is then steadily increasing up to 1.8 due to the lack of (very) high elevation satellites near the poles. This latitudinal behavior is static as it mainly originates from the inclination of the satellite orbits. The longitudinal fluctuations seen in the plot (e.g., Indian Ocean, Pacific, and Sahara) on the other hand vary slowly over time with the orbit position of the satellites and the Earth's rotation.

Another relevant aspect in terms of the geometric influence is the deterioration of the DOPs with a raising elevation mask from the currently used value of $5^{\circ}$. The benefits of increasing the elevation mask from an operational point of view will be further discussed at the end of Section 5.5. Here, we only focus on the geometrical implications.

In Figure 3 DOP values are simulated every 10 minutes within 10 days using GPS single constellation for an elevation mask between $1^{\circ}$ and $25^{\circ}$. Figure 3(a) shows the dependency of the different DOP terms for a single location (Braunschweig, Germany) and Figure 3(b) focuses on the VDOP at different latitudes.

In Figure 3(a) all the different DOPs are normalized by their value at $5^{\circ}$ elevation mask (accordingly the value at $5^{\circ}$ is 1 for all of them). One interesting fact to mention here is that the VDOP is more vulnerable to elevation masking compared to the horizontal DOP. At about $22^{\circ}$ elevation mask the initial VDOP doubled, while the ratio stays below 1.5 for more realistic potential masking angles of up to $15^{\circ}$. From these results an increase of the resulting vertical protection levels of roughly $18 \%$ and $44 \%$ can be anticipated when going from $5^{\circ}$ to $10^{\circ}$ or 5 to $15^{\circ}$ elevation mask in GPS. Actual protection level simulations will investigate this further in Section 5.5. In Figure 3(b) we show the dependency on the geographic latitude. As we have already seen in the global plot of VDOPs (Figure 2) the properties of the satellite orbits lead to a latitudinal profile. With the different elevation distributions of satellites seen from different latitudes it can be expected that the vulnerability to elevation masking is varying as well. When looking at Figure 3(b) we see that this effect is visible but not as pronounced as maybe expected. In accordance with the global VDOP profile we can see the VDOP more susceptible at very high latitudes than at medium latitudes, but larger differences show up only for hardly relevant elevation masks greater than $15^{\circ}$.

4.1.2. Satellite Selection. Using all satellites in view is the most common approach when it comes to navigation in a single constellation scenario. Nevertheless, there are some reasons why a limitation of the number of used satellites can be desirable as soon as a larger number of satellites are available to choose from. This can be a limited number of channels in a receiver, capacity limitations on the GBAS data link, or a limited processing capability like in case of unmanned aircraft systems (UAS). In previous work we already presented a feasible approach for the selection of optimized satellite subsets [12]. This method utilizes information from an all-inview solution to find fixed-size subsets in real time, orders of magnitude faster than an optimal selection by bruteforce. It was found that the magnitude of the projection factors in the $\mathbf{S}$ matrix shows a strong correlation with the probability of a satellite being part of an optimal subset. Taking this into account an iterative selection process can be significantly sped up. The selected subconstellations only show an average increase of the protection levels of less than $1 \%$. This selection algorithm will be utilized in some of the simulations, to compare DOPs and protection levels of all-in-view with limited subsets out of dual constellation scenarios. When referring to "best 14" subsets in this paper we always address this quasi-optimal solution which we expect to be sufficiently close to the real "optimum," that is, the smallest achievable protection levels. Except from avoiding impractically long simulation times this also considers the fact that perfect selection might not always be possible in real time applications. The shown best- $N$ results can thus be seen as conservative examples of what can at least be achieved with limited subsets.

4.1.3. Improvements from a Second Constellation. To complete the geometric considerations and come back to the initial question, we will now investigate the actual improvement (in terms of DOP) of adding a second constellation. We started with Figure 2 which provided a global overview of average VDOPs. In relation to this, Figure 4 shows how the VDOP values decrease for a dual constellation scenario. On the global map the ratio between single and dual constellation VDOP is plotted on the same 2-by-2-degree grid as before. Figure 4(a) illustrates the comparison when using all satellites in view. As we can see the values decrease by $25-40 \%$ which roughly translates into accordingly improved vertical navigation performance. For most regions the VDOP is as low as 0.65 to 0.7 times the value of GPS single constellation. 


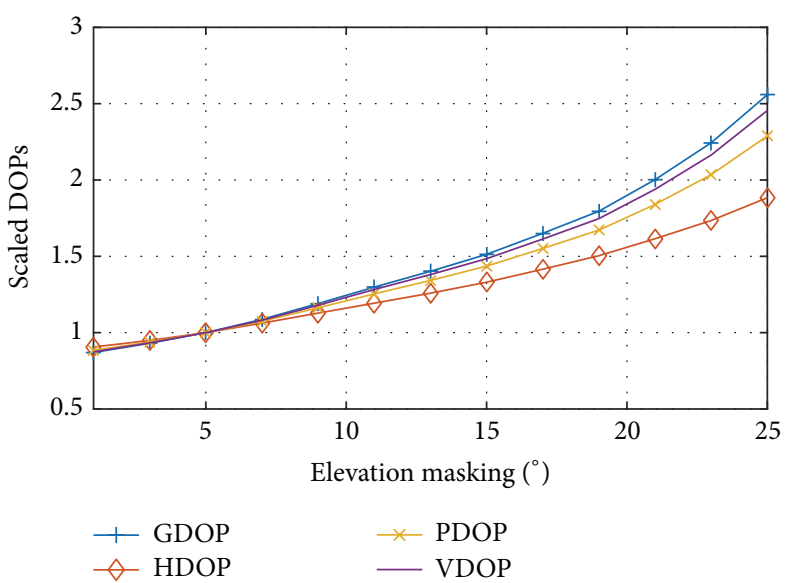

(a) Ratio between DOPs over elevation mask and DOPs for an elevation mask of $5^{\circ}$ in Braunschweig

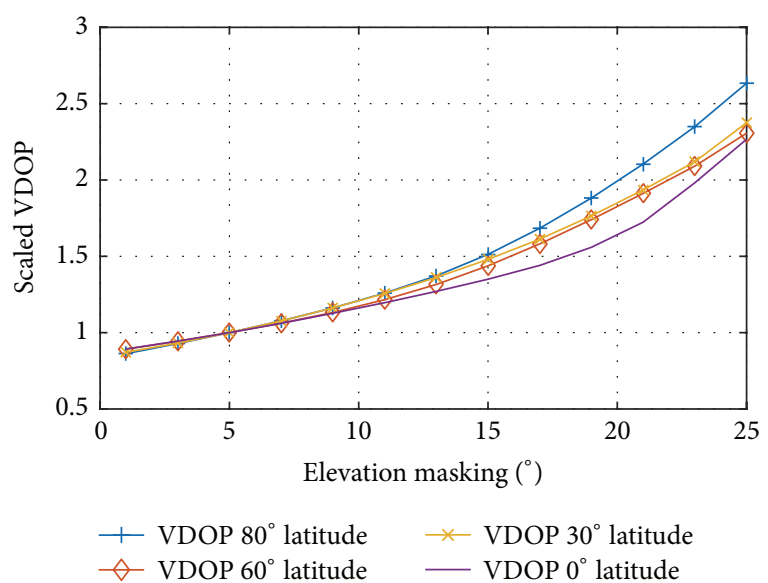

(b) Ratio between VDOP over elevation mask and VDOP with $5^{\circ}$ elevation mask for different latitudes

FIGURE 3: Influence of elevation masking on DOP values.

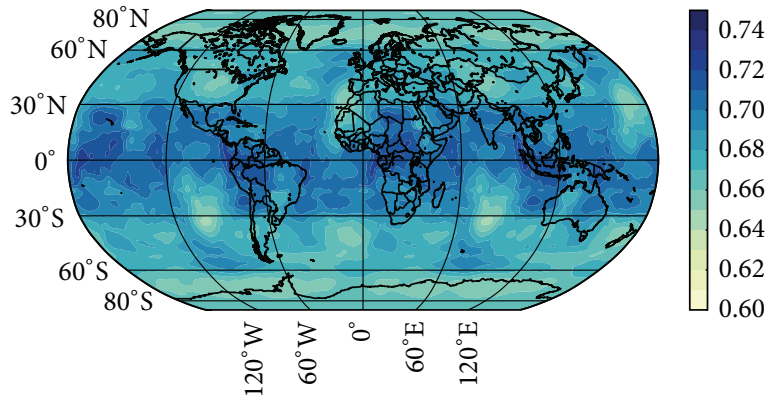

(a) Dual constellation, all-in-view

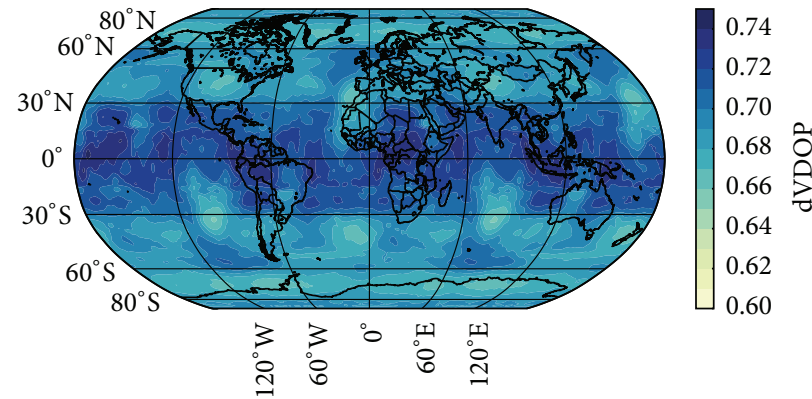

(b) Dual constellation, best 14 heuristically selected

FIGURE 4: Decrease of VDOP in comparison to single constellation GPS ( $5^{\circ}$ elevation mask).

Particularly regions with unfavorable geometry (e.g., poles and irregularities from Figure 2) with GPS alone benefit most from the second constellation.

When comparing the all-in-view results with the results of limited subsets (best 14 satellites) in Figure 4(b) we find a similar picture. As expected, the DOPs increase slightly when using only 14 satellites instead of up to 26 which are possibly available. Nevertheless the average DOPs are still only about 0.7 times as big as in the single constellation case and increase only a few percent ( $1 \%$ to $4 \%$ ) compared to using all-in-view. Later on in the performance simulations we will see how this minor deterioration translates into actual protection levels.

4.2. Overview of Sigma Contributions. Until now we discussed the influence of the geometry on the protection levels. The other component that drives the protection level is the residual uncertainty of the differential error. As defined in (7) the standard deviation of the residual uncertainty of the differential GBAS error $\sigma_{i}$ consists of the root sum square of uncertainties introduced through ionospheric and tropospheric decorrelation as well as the contribution of the ground and airborne multipath and noise. In order to evaluate the performance of new processing modes, each individual sigma has to be reevaluated as they differ for each technique. The ionospheric uncertainty is only relevant for the single frequency processing, whereas the tropospheric uncertainty is not frequency dependent and thus persists for all processing schemes. Both uncertainties become smaller as the aircraft approaches the destination airport or GBAS station. On the other side, the receiver noise and multipath from the ground station remain the same for all aircraft positions. In the following subsections we discuss each sigma individually and the changes that have to be taken into account for the different processing modes.

4.2.1. Sigma Ground. The ground broadcasts values for $\sigma_{\text {gnd }}$ associated with the pseudorange correction for each satellite and each service type. These broadcast values are derived from at least 24 hours of measurements from the ground station and represent the actual local system characteristics. The ground message contains the values for $\sigma_{\text {gnd }}$ computed based on a 100-second smoothing time constant (used in GAST C positioning and error bounding and GAST D for error bounding) and a 30-second smoothing time constant (used in GAST D positioning). The multipath and noise are partly dependent on the signal modulation and are thus different 


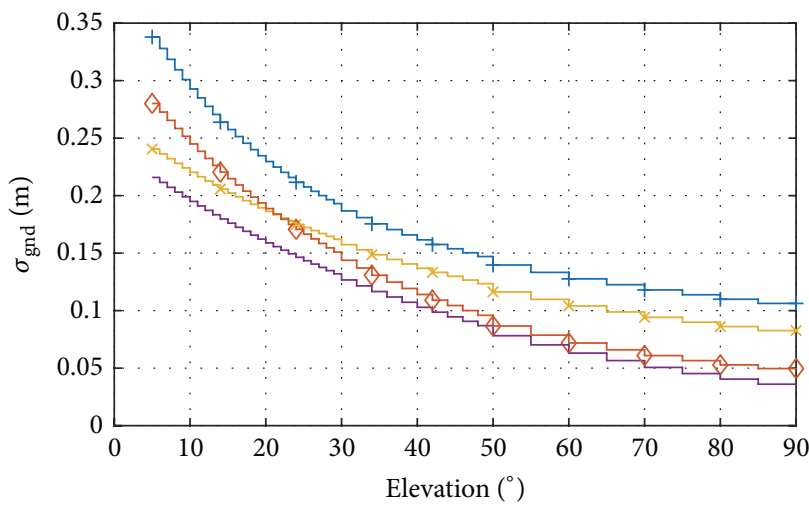

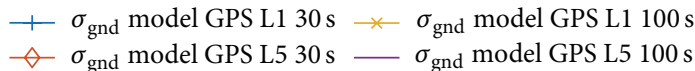

(a) Models used for GPS

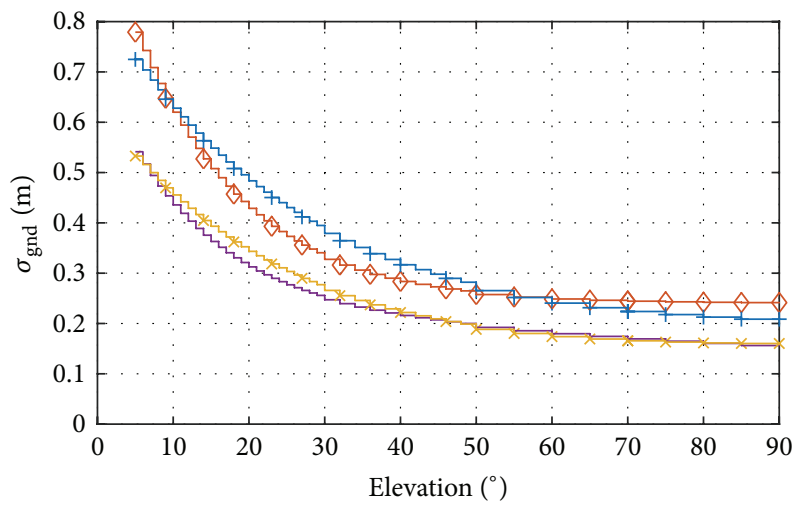

$\leftarrow \sigma_{\text {gnd }}$ model GPS Ifree $30 \mathrm{~s} \leftarrow \sigma_{\text {gnd }}$ model GPS Ifree $100 \mathrm{~s}$

$\diamond \sigma_{\text {gnd }}$ model Gal Ifree $30 \mathrm{~s} \_\sigma_{\text {gnd }}$ model Gal Ifree $100 \mathrm{~s}$

(c) Ifree models for GPS and Galileo

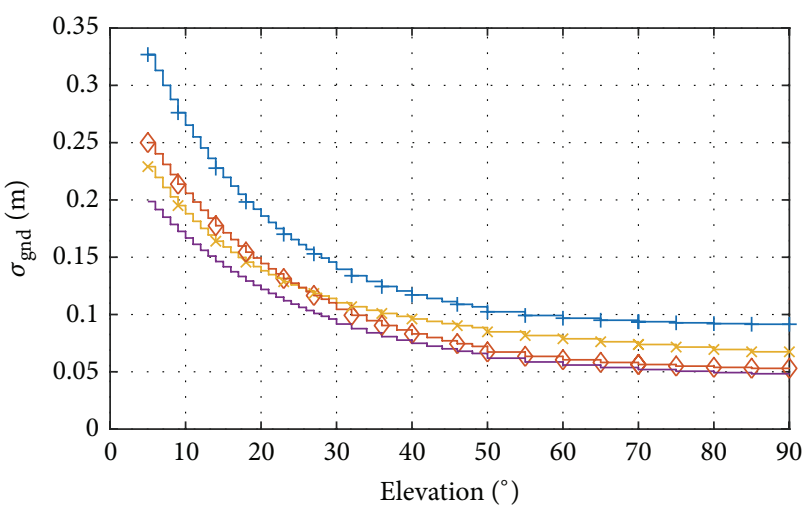

$\leftarrow \sigma_{\text {gnd }}$ model Gal E1 30s $\leftarrow \sigma_{\text {gnd }}$ model Gal E1 $100 \mathrm{~s}$ $\diamond \sigma_{\text {gnd }}$ model Gal E5 30s $\_\sigma_{\text {gnd }}$ model Gal E5 $100 \mathrm{~s}$

(b) Models used for Galileo

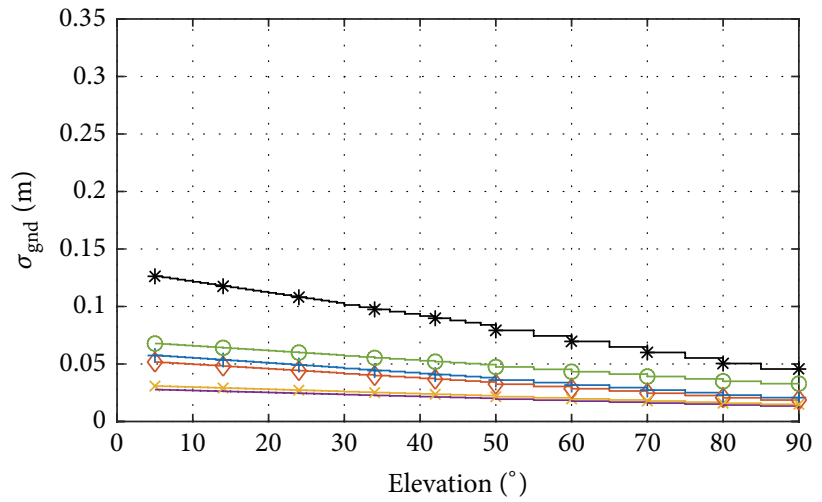

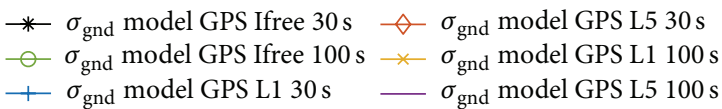

(d) GPS models for MLA antennas

FIGURE 5: $\sigma_{\text {gnd }}$ curves for different processing schemes.

for different signals. In [13] we evaluated the $\sigma_{\text {gnd }}$ models for the new available L5 signals broadcast by the GPS Block IIF satellites and E1 as well as E5a signals broadcast by Galileo satellites. We use these results obtained from measurements to derive the models for the protection level simulations presented in the next section. The measurements used were taken from DLR's GBAS test bed located in Braunschweig in northern Germany. The test bed uses Leica AR-25 choke ring antennas and Javad Delta 3 receivers. We derived models for all signals for both smoothing time constants. The results show that the noise and multipath level of the Galileo signals and of the GPS L5 signals is smaller than that of GPS L1. The difference between the GPS L1 and GPS L5 $\sigma_{\text {gnd }}$ models for 30 - and 100-second smoothing is shown in Figure 5(a). The reduction of the smoothing time constant brings a significant increase of the residual noise and multipath, especially on the GPS L1 signals. This difference is less pronounced for the L5 signals since the higher transmitted power and higher chipping rate yield an improved resistance to multipath. A similar comparison is presented in Figure 5(b) for the Galileo
E1 and E5a signals. Both models show a slight improvement compared with the GPS ones and the difference between 30 and 100 seconds is again more pronounced on the E1 signal. Due to the combination of two code measurements, the Ifree solution leads to significantly higher multipath and noise. This increase is clearly visible in Figure 5(c) where the Ifree curves for $\sigma_{\text {gnd }}$ GPS (L1/L5) and Galileo (E1/E5a) are shown for both smoothing time constants. All $\sigma_{\text {gnd }}$ values were computed by sorting data into elevation bins with bin sizes of $1^{\circ}$ up to a satellite elevation of $30^{\circ}, 2^{\circ}$ between $30^{\circ}$ and $50^{\circ}$, and $5^{\circ}$ for all higher elevations.

Even though the antenna type and the site where it is installed do not fulfill the requirements for an operational GBAS, the results give a good indication of what can be expected from the different processing modes. In Figure 5(d) we show the $\sigma_{\text {gnd }}$ curves for a MLA antenna versus elevation for all processing modes. The GPS L1 C/A curves (100 seconds and 30 seconds) were derived from measurements collected with a MLA antenna. In order to derive the curves for all signals we applied the ratios between signals that we obtained 
from our measurements. Thus these curves do not represent actual data but simulations in these cases. They are not representative for a future MLA performance but are used to assess the lower noise and multipath impact on the protection level.

4.2.2. Sigma Air. The $\sigma_{\text {air }}$-term represents the residual uncertainty attributed to the residual airborne multipath and thermal noise after carrier smoothing. Unlike using actual measured performance as for the ground contribution, $\sigma_{\text {air }}$ is described based on standardized error models. These models have to be conservative in order to cover all aircraft installations with sufficient confidence.

As no multipath limiting antennas and no siting away from reflecting surfaces are possible on the fuselage of an aircraft the airborne multipath contributions are larger than the contribution from ground. In the vicinity of the airport it usually is the largest contributor to the protection level.

For single frequency GBAS, GPS L1 measurements standard models called Airborne Accuracy Designators (AAD) were presented in [14] considering different airborne receivers. Two types of designators for the different receiver thermal noise were proposed: AAD-A and AAD-B with their performance models shown in (16). The adopted multipath model as in the SARPs [11] is given in (15).

The total $\sigma_{\text {air }}$ is the root sum square of the multipath and noise components as function of satellite elevation, shown in (17). Consider

$$
\begin{aligned}
\sigma_{\mathrm{MP}}(\theta) & =0.13+0.53 \cdot e^{-\theta / 10}, \\
\sigma_{n}(\theta) & =0.15+0.43 \cdot e^{-\theta / 6.9}, \text { for AAD-A, } \\
\sigma_{n}(\theta) & =0.11+0.13 \cdot e^{-\theta / 4}, \text { for AAD-B, } \\
\sigma_{\text {air }}(\theta) & =\sqrt{\sigma_{\mathrm{MP}}^{2}(\theta)+\sigma_{n}^{2}(\theta) .}
\end{aligned}
$$

These models were defined only for 100-second smoothing. As previously mentioned, the GAST D position solution is based on 30-second smoothed pseudoranges, while for error bounding the models based on 100-second smoothing are used. The difference in noise is accounted for in $D_{V}$ and $D_{L}$ (6).

However, there are no airborne models defined for the new GPS L5 signals and Galileo signals. Using measurements collected during our flight tests we investigated the airborne performance of the new signals. The preliminary $\sigma_{\text {air }}$ curves for these new signals are presented in [15]. The amount of data is still very limited and we can not derive final models, but they give a first indication of the difference between different signals. The results considered in this work are derived from data collected from DLR's Airbus A320, as it is representative of a commercial airplane. The airborne receiver has a $23 \mathrm{MHz}$ bandwidth, and the correlation peak sampling values were set to 0.1 chips for L1/E1 signals and 1.0 chip for L5/E5a signals. Figure 6(a) shows the comparison of the $\sigma_{\text {air }}$ curve derived from measurements using a 100second smoothing time constant (red pluses) with the AADs curves. The curves are plotted versus satellite elevation in order to be consistent with the AAD definition. The worse L1 performance compared with the AAD-B curve may be attributed to the performance of the multiband antenna installed on the aircraft. The blue curve (diamonds) shows the measurements-based curve for GPS L1 using 30-second smoothing time constant. Compared with 100 seconds this curve is larger as the airborne measurements are affected by fast changing short-range multipath. Based on the curves obtained from the measurements we derived two model curves (a more conservative and a somewhat optimistic one) for each signal. For the development of final models and realistic overbounds more data is required. However, for the scope of this paper it was only necessary to have consistent models representing the data available so far to make performance results comparable. These models are used in the protection level simulations and the impact of the different models on the protection levels will be shown in Section 5.5, Figure 15(b). As an example Figure 6(b) shows the GPS 30-second curve obtained from measurements again (black curve) and the two models derived and used in the simulations. The GPS L5 and Galileo E5a signals that have a ten times higher chipping rate than the L1 and E1 signals show again an improved performance in terms of multipath. Unlike on the ground, on the airborne side the two signals show comparable performance; thus one common model was adopted for this study. Figure 6(d) shows the $\sigma_{\text {air }}$ curves for GPS L5 and Galileo E5a for 100-second smoothing time constant and the models derived from the measurements. The Galileo E1 signals show similar performance compared with GPS L1. In Figure 6(c) we show in the same manner the measurement-based curve and the two models considered for Galileo E1 using a 30-second smoothing time constant. Note again that these models are to be considered preliminary and they will have to be validated as soon as more data is available. In a similar manner we derived the curves also for all other signals. The complete set of models is shown in Figure 17, together with the fitting equations in Table 1.

4.2.3. Sigma Ionosphere. We are now going to discuss the residual ionospheric error described again by a zero-mean Gaussian distributed random variable with standard deviation $\sigma_{\text {iono }}$.

For single frequency smoothing $\mathrm{L} 1 \sigma_{\text {iono }}$ is defined in Section 2.3.1.2.3 of the MOPS [7] as in (18). $F_{p p}$ is the verticalto-slant obliquity factor, $\sigma_{\text {vig }}$ the standard deviation of nominal ionospheric uncertainty due to the spatial decorrelation, $x_{\text {air }}$ the 2D horizontal distance between ground station and user, $\tau$ the smoothing time constant, and $v_{\text {air }}$ the ground speed of the aircraft:

$$
\sigma_{\text {iono }}=F_{p p} \cdot \sigma_{\text {vig }} \cdot\left(x_{\text {air }}+2 \tau \cdot v_{\text {air }}\right) .
$$

Unlike $\sigma_{\text {air }}$ and $\sigma_{\text {gnd }}$, the $\sigma_{\text {iono }}$ term is depending on the distance between user and the ground station and also with the speed of the aircraft representing the decorrelation when moving through an ionospheric gradient. The term $2 \tau v_{\text {air }}$ represents the additional error introduced by single frequency smoothing due to the ionospheric divergence created by an aircraft moving through a spatial ionospheric 


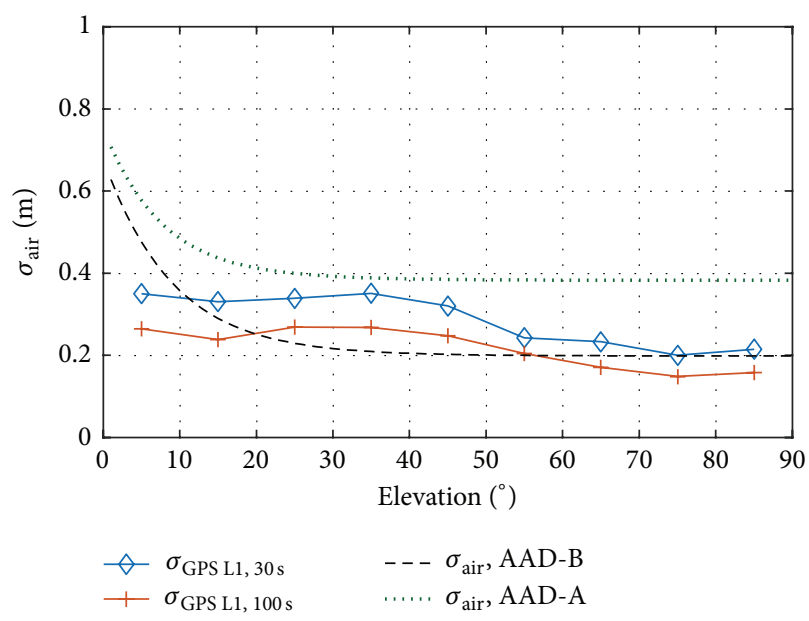

(a) Comparison of AAD to models from own measurements

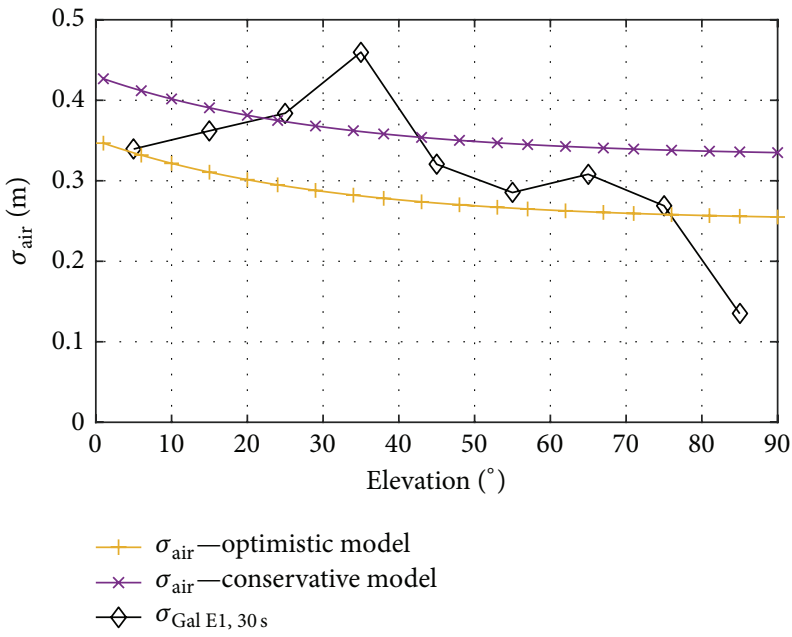

(c) Galileo E1 $30 \mathrm{~s}$

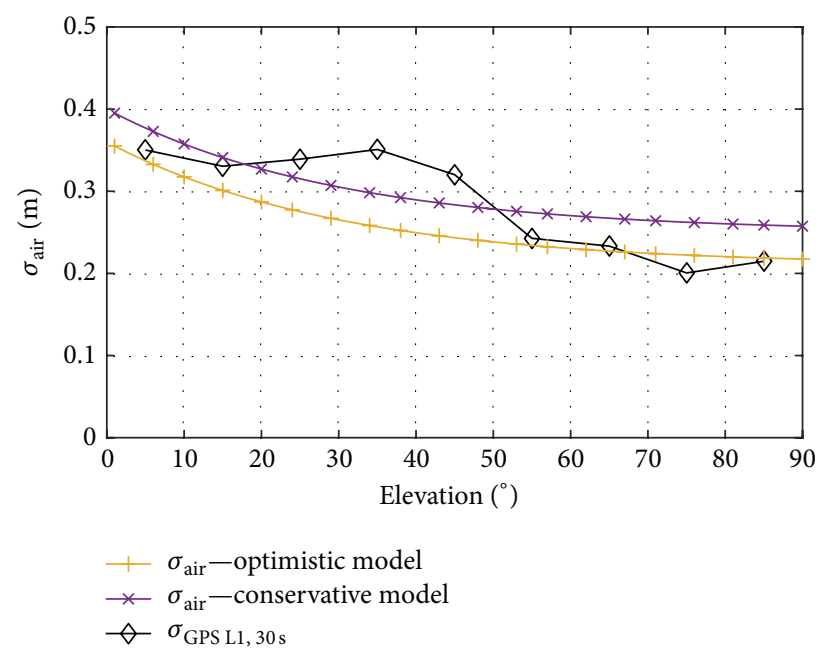

(b) GPS L1 $30 \mathrm{~s}$

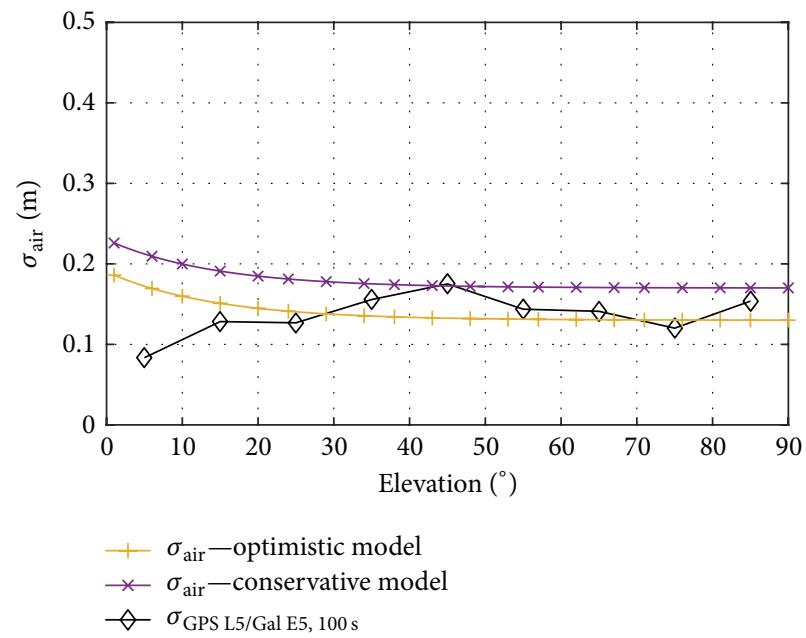

(d) GPS L5/Galileo E5a $100 \mathrm{~s}$

FIGURE 6: $\sigma_{\text {air }}$ models for different processing schemes.

gradient with velocity $v_{\text {air }}$. The ground station broadcasts a bounding value of $\sigma_{\text {vig }}$ to the aircraft that computes $\sigma_{\text {iono }}$ based on the speed and distance to the ground station. As in GAST C the ground is responsible for protection of the airborne user from potentially large ionospheric gradients, the broadcast $\sigma_{\text {vig }}$ may be inflated in order to make vulnerable satellite geometries unavailable [16]. For GAST D the ground and airborne systems share the responsibility to ensure that no large differential errors can occur without being detected. Additional monitoring is used making the inflation unnecessary. However, $\sigma_{\text {vig }}$ depends on the region where the GBAS is located and frequency of the navigation signal. For L1 a value of $4 \mathrm{~mm} / \mathrm{km}$ was derived for the CONUS (contiguous United States) region [16] while $2.07 \mathrm{~mm} / \mathrm{km}$ was the value derived for Germany [2]. On L5/E5a the ionospheric delay increases by a factor of 1.8 as shown in (19), where $I_{\mathrm{L} 1}$ is the ionospheric delay on $\mathrm{L} 1, I_{\mathrm{L} 5}$ is the ionospheric delay on $\mathrm{L} 5 / \mathrm{E} 5 \mathrm{a}$, and the frequencies $f_{\mathrm{L} 1}$ and $f_{\mathrm{L} 5}$ are $1575.42 \mathrm{MHz}$ and 1176.45 MHz, respectively. As the nominal decorrelation is a physical property of the ionosphere and the electron density $\sigma_{\text {iono }}$ for L5 is about 1.8 times larger than for L1:

$$
I_{\mathrm{L} 1} f_{\mathrm{L} 1}^{2}=I_{\mathrm{L} 5} f_{\mathrm{L} 5}^{2} \Longrightarrow I_{\mathrm{L} 5}=\frac{f_{\mathrm{L} 1}^{2}}{f_{\mathrm{L} 5}^{2}} I_{\mathrm{L} 1} .
$$

The Ifree dual frequency combination removes the relevant ionospheric delay completely at both ground and airborne receivers. Thus, no ionosphere-related errors remain to be considered and the corresponding $\sigma_{\text {iono }}$ is set to 0 .

4.2.4. Sigma Troposphere. The $\sigma_{\text {tropo }}$ contribution represents the standard deviation of the residual uncertainty introduced by the tropospheric decorrelation between the aircraft and the ground station. The tropospheric error is not frequency dependent and $\sigma_{\text {tropo }}$ thus persists for all processing schemes (single frequency L1, single frequency L5, and dual frequency Ifree). The tropospheric residual uncertainty depends on the atmospheric conditions and on the height of the approaching 
TABLE 1: Conservative (right) and optimistic models for $\sigma_{\text {air }}$.

\begin{tabular}{lcc}
\hline Model & Fitting equation $(\mathrm{s})$ \\
\hline AAD-A & $\sqrt{0.36^{2}+\left(0.13+0.53 \cdot e^{-\theta / 10}\right)^{2}}$ \\
AAD-B & $\sqrt{0.15^{2}+\left(0.13+0.53 \cdot e^{-\theta / 10}\right)^{2}}$ \\
\hline GPS L1 30 s & $0.21+0.15 \cdot e^{-\theta / 30}$ & $0.25+0.15 \cdot e^{-\theta / 30}$ \\
GPS L1 100 s & $0.16+0.13 \cdot e^{-\theta / 30}$ & $0.20+0.18 \cdot e^{-\theta / 20}$ \\
Galileo E1 30 s & $0.25+0.1 \cdot e^{-\theta / 30}$ & $0.33+0.1 \cdot e^{-\theta / 30}$ \\
Galileo E1 100 s & $0.14+0.07 \cdot e^{-\theta / 14.3}$ & $0.18+0.07 \cdot e^{-\theta / 14.3}$ \\
\hline GPS L5/Galileo E5a 30s & $0.16+0.06 \cdot e^{-\theta / 14.3}$ & $0.20+0.06 \cdot e^{-\theta / 14.3}$ \\
GPS L5/Galileo E5a 100 s & $0.13+0.06 \cdot e^{-\theta / 14.3}$ & $0.17+0.06 \cdot e^{-\theta / 14.3}$ \\
\hline GPS Ifree 30 s & $0.4+0.4 \cdot e^{-\theta / 33.3}$ & $0.6+0.4 \cdot e^{-\theta / 33.3}$ \\
GPS Ifree 100 s & $0.25+0.35 \cdot e^{-\theta / 50}$ & $0.35+0.45 \cdot e^{-\theta / 50}$ \\
Galileo Ifree 30 s & $0.4+0.25 \cdot e^{-\theta / 33.3}$ & $0.6+0.25 \cdot e^{-\theta / 33.3}$ \\
Galileo Ifree 100 s & $0.32+0.2 \cdot e^{-\theta / 50}$ & $0.4+0.2 \cdot e^{-\theta / 50}$ \\
\hline
\end{tabular}

aircraft (relative to the airport). Thus, $\sigma_{\text {tropo }}$ becomes very small in the last part of the approach. It is defined for L1 in DO-253 standards [7] as

$$
\sigma_{\text {tropo }}=\sigma_{n} h_{0} \frac{10^{-6}}{\sqrt{0.002+\sin (\theta)^{2}}}\left(1-e^{-\Delta h / h_{0}}\right),
$$

where $\sigma_{n}$ is the refractivity uncertainty transmitted by the ground system (unitless), $h_{0}$ the tropospheric scale height transmitted as well, $\theta$ the elevation angle of the satellite, and $\Delta h$ the height of the aircraft above the GBAS reference point (in meters). The ground system is responsible for broadcasting the parameters $\sigma_{n}$ and $h_{0}$ which should be consistent with prevailing conditions at the site. These parameters may be set to constant values that cover the worst case condition expected during operation [14]. For our case we use $\sigma_{n}=20$ and $h_{0}=7949.42 \mathrm{~m} \mathrm{[6]}$.

4.2.5. Other Effects. Another effect that will have to be considered is the interfrequency bias that affects the dual frequency Ifree processing. It results from the satellite hardware and may not be negligible. It furthermore differs between satellites and the effect on the user equipment depends on receiver hardware and tracking implementations. However it might be possible to characterize this effect on a persatellite basis and correct it accordingly such that only a small residual error uncertainty has to be accounted for [13]. In this study we do not take this effect into account as there is no clear solution yet on how to handle this matter.

\section{Performance Simulations with Different Assumptions}

After the previous discussion of the parameters that influence the protection levels we will now show the actual results of the overall performance simulations. We start off by defining the general setup used for the simulations. Afterwards we show a global map, as we already did for the DOPs, just to give a general idea of the VPL spread on a global scale. As the final simulations are (for practical reasons) carried out only for a single location, we will continue with a study on transferability of the results to other locations. Finally we show results for many different possible GBAS architectures in order to illustrate the implications of selecting a specific mode.

In order to compare the performance of the different processing modes considered we perform time-wise VPL averaging. The mean value is not necessarily representative for all the situations and all the locations, but it gives good insights about the general behavior of the different processing modes. Furthermore, the exact performance of the protection levels is very location dependent and specific to each GBAS installation, for example, due to selective masking according to local surroundings.

5.1. General Setup. The general assumptions for all the following simulations are as follows:

Location of the GBAS reference station at Braunschweig Airport $\left(52^{\circ} 19^{\prime} 9^{\prime \prime} \mathrm{N}, 10^{\circ} 33^{\prime} 32^{\prime \prime} \mathrm{E}\right)$.

$5^{\circ}$ elevation mask for both GPS and Galileo.

Velocity of the aircraft according to speed profile [17].

Height of the aircraft which follows a $3^{\circ}$ glide path angle up to $3050 \mathrm{~m}(10000 \mathrm{ft})$.

$\sigma_{\text {gnd }}$ curves according to the models in Figure 5.

$\sigma_{\text {air }}$ according to the conservative models from Figure 6 as well as Figure 17.

$\sigma_{\text {vig }}$ equal to $4 \mathrm{~mm} / \mathrm{km}$.

No geometry screening and thus no sigma inflation.

Plots showing mean vertical protection levels averaged over 5000 samples during 10 days.

$5 \mathrm{~km}$ distance to the GBAS reference.

Simulations over distance (to the reference) assuming the same fixed satellite geometry for all distances.

Simulations over distance starting at $x_{\text {air }}=0$.

Only $\mathrm{H} 0$ protection levels which are simulated. Faults and ephemeris PLs are not considered in this study.

Whenever exceptions to this setup are made or parameters are varied it is stated in the according section. In terms of the last bullet point it has to be mentioned that at larger distances to the airport in general the ephemeris protection levels (which are not considered here) dominate. As they depend on the station performance, the setup, and also the used ephemeris monitoring method, it was not feasible to introduce them in this study in a representative way. Thus they are intended to be investigated further for the dual constellation case in a separate study. The used profiles for speed as well as for height are additionally illustrated in Figure 7. For the speed profile we assumed the medium model of the assumptions from [17]. In fact the actual speed and height profile during landing can vary greatly depending on the airplane type, wind and weather conditions, traffic, and so on. Nevertheless it is reasonable to assume some kind of 


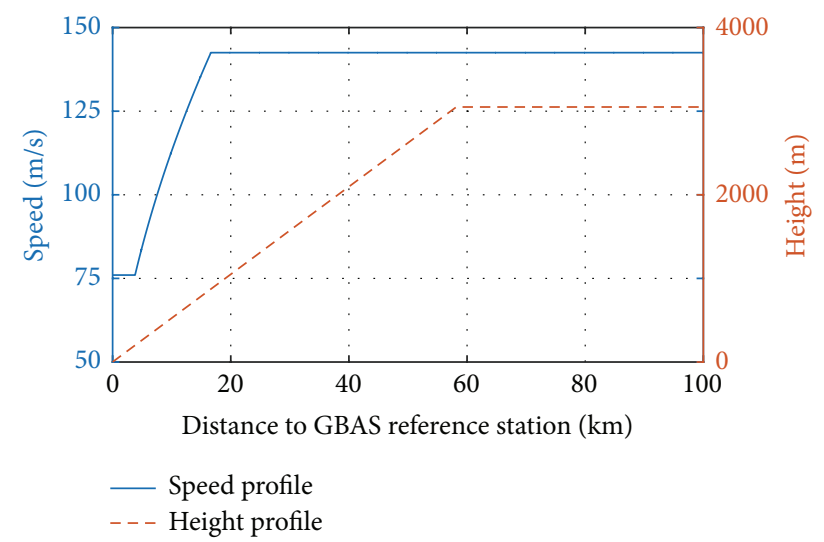

FIGURE 7: Speed and height profile used for protection level simulations.

deceleration as well as descent during final approach. The specific shape might change and alter the protection levels slightly, but the general behavior is going to stay similar.

5.2. Global Maps. To first give a general idea how the nominal protection levels globally look like we can take a look at Figure 8(a). In this case we plotted the mean VPL at $5 \mathrm{~km}$ distance to the GBAS reference for current GAST D processing. Unsurprisingly we can recognize the same latitudinal distribution with few fluctuations in medium latitudes and decreasing performance close to the poles as before for the VDOPs. As the sigma terms are basically independent of the location (except for ionospheric and tropospheric parameters for some extent) we predominantly see a scaled version of the DOP map.

Figure 8(b) shows exemplary how the daily variations for one location look like for some of the afterwards discussed processing modes. Interesting to see are not only the in general lower VPLs but also the greatly reduced variations over time when adding a second constellation (yellow crosses). Even though the mean VPL for GPS GAST D (green circles) is still lower compared to the dual constellation Ifree solution (violet line), we see that the maximum (around 3:00 in the plot) already extends almost as high. This is already true at $5 \mathrm{~km}$ distance to the airport and gets even more pronounced when going further away.

The comparison in Figure 9 gives another good hint on how a second constellation makes the protection levels more regular and predictable. For this illustration the protection levels which are simulated on a global grid as in Figure 8(a) are stacked along longitudes. Basically as one would view the resulting "protection level surface" from one side along latitudes. On the left in Figure 9(a) we compare mean and 5th and 95th percentile. Particularly between $10^{\circ}$ and $40^{\circ}$ it is obvious from the 95th percentiles how large the fluctuations along longitudes are when using only GPS.

When we now compare this to Figure $9(\mathrm{~b})$ on the right, the improvement with a second constellation is clearly visible. Plot (2) is the same 95th percentile for GAST D as on the left. Additionally we plotted the VPLs of two dual constellation modes. Comparing the single frequency L1E1 mode (3) with GAST D we can find the average improvement to 0.6 to 0.7 times the original protection level in analogy to Figure 4(a) (as the sigmas are comparable in this case). Even more important the longitudinal variance disappears almost completely. While in case of single constellation GAST D the spread ranges up to $1.5 \mathrm{~m}$ it stays below $0.25 \mathrm{~m}$ in (3) for DC L1E1. Plot (1) in the same figure shows the influence of the increased Ifree sigmas in the dual constellation scenario.

5.3. Comparability and Transferability of the Results. One major issue when targeting performance comparisons in terms of GBAS protection levels is the basically unlimited amount of possible setups considering, for example, all processing modes at all current airports worldwide or similar. Even after simulating everything it is impossible to show results for all cases in a reasonable manner. As we perform our final performance comparisons for one airport it is interesting to study how meaningful results for one specific location are in terms of general conclusions. In Figure 10 we compare normalized VPLs for two processing modes at different latitudes (longitude is always $0^{\circ}$ ). The normalization is done by dividing all VPL values by the VPL at $0 \mathrm{~km}$ distance to the GBAS reference (thus all plots start at value 1). When we compare especially the plots for $0^{\circ}, 30^{\circ}$, and $55^{\circ}$ latitude we see a very similar behavior with a divergence of less than $4 \%$. Only at very high latitudes the results start to diverge more, but for practical GBAS applications latitudes above $70^{\circ}$ are of minor relevance anyway (due to lack of airports). Actual protection levels at different locations are according to this quite similar in terms of qualitative behavior, only scaled depending on the location dependent geometry (i.e., DOP values).

Additionally, as we are going to show only mean VPLs in all the following plots, Figure 11 relates this to minimum, maximum, and 95th percentile VPL values. In case of single constellation (exemplary for GPS L5 $30 \mathrm{~s}$ in Figure 11(a)) the maximum VPLs exceed the mean value by about $60 \%$ while the minimum is about $30 \%$ smaller than the mean. For dual constellation modes we can again notice the smaller spread with a maximum about $26 \%$ larger than the mean value and a $20 \%$ smaller minimum. As these ratios are predominantly geometry dependent they can be roughly transferred to all the mean VPL plots in the following sections.

5.4. VPL Contributions. As we have seen from Section 5.3 the average achieved protection levels are mainly scaled by the location but not significantly altered. Thus all further simulations are carried out for one location, namely, the Braunschweig Airport as mentioned in Section 5.1.

Figure 12 shows simulated protection levels as a function of distance between the GBAS reference point and an airborne user. Different shadings in the plots show the different contributions to the protection levels. Shown in red on the bottom is always the contribution for $\sigma_{\text {gnd }}$ followed by the yellow area indicating the $\sigma_{\text {air }}$ contribution. The small blue area above shows the contribution of $\sigma_{\text {tropo }}$ and for the single frequency cases in subplots (a)-(d) the green area on top 


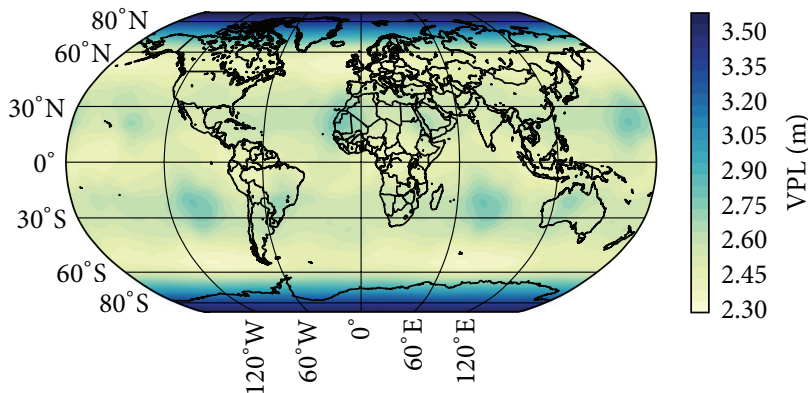

(a) Average global H0 VPL for GAST D

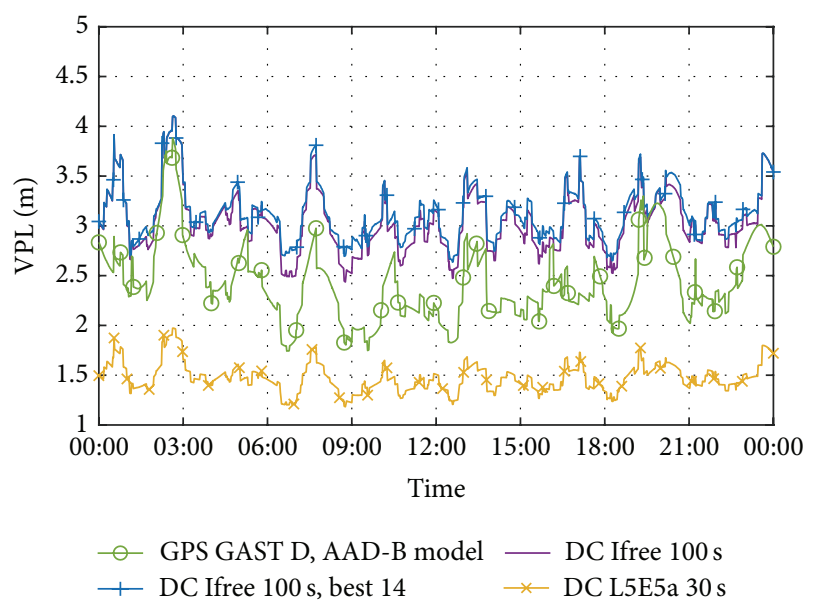

(b) Vertical protection levels for different processing modes over time in Braunschweig

FIgURE 8: Global VPL for GAST D and exemplary comparison plot over time.

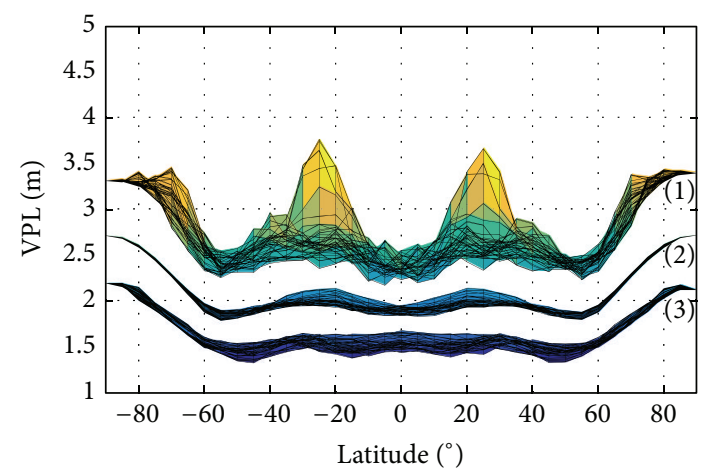

(a) 95 th percentile (1), mean value (2), and 5th percentile (3) for GAST

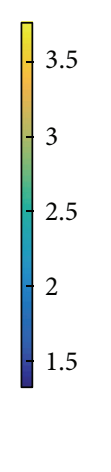

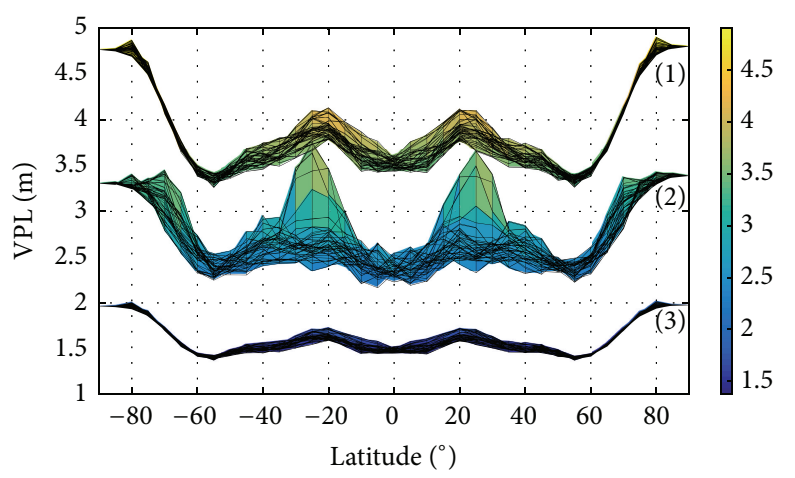

(b) 95th percentiles for DC Ifree $100 \mathrm{~s}(1)$, GAST D (2), and DC L1E1 $100 \mathrm{~s}(3)$

FigURE 9: Global H0 VPLs stacked along longitudes. Vertical extents at each latitude represent the variation along longitudes.

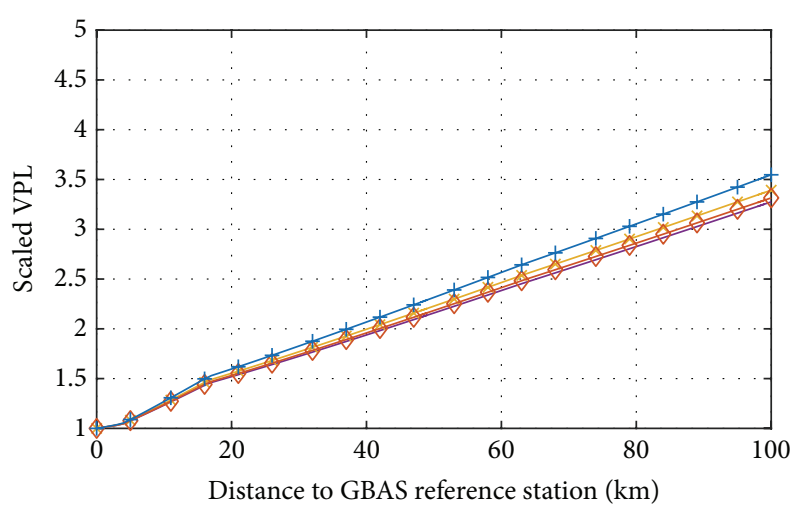

十 DC L1E1100s@80 latitude $\rightarrow$ DC L1E1 $100 \mathrm{~s} @ 30^{\circ}$ latitude

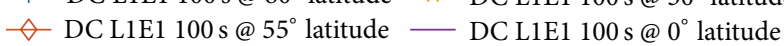

(a) For dual constellation L1E1 $100 \mathrm{~s}$

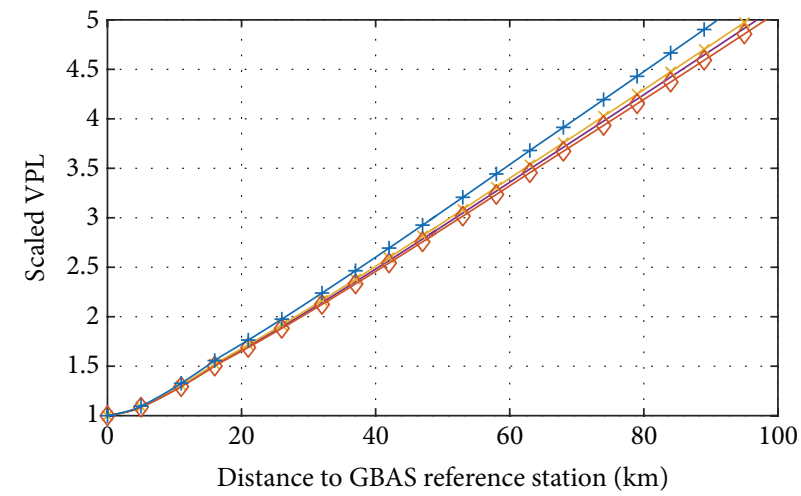

+ GPS L5 30s @ 80 latitude $\rightarrow$ GPS L5 30s@ 30 latitude $\prec$ GPS L5 30s@5 $55^{\circ}$ latitude — GPS L5 30s @ $0^{\circ}$ latitude

(b) For single constellation L5 $30 \mathrm{~s}$

FIGURE 10: H0 VPL behavior at different latitudes, normalized by VPL at zero distance to the airport. 


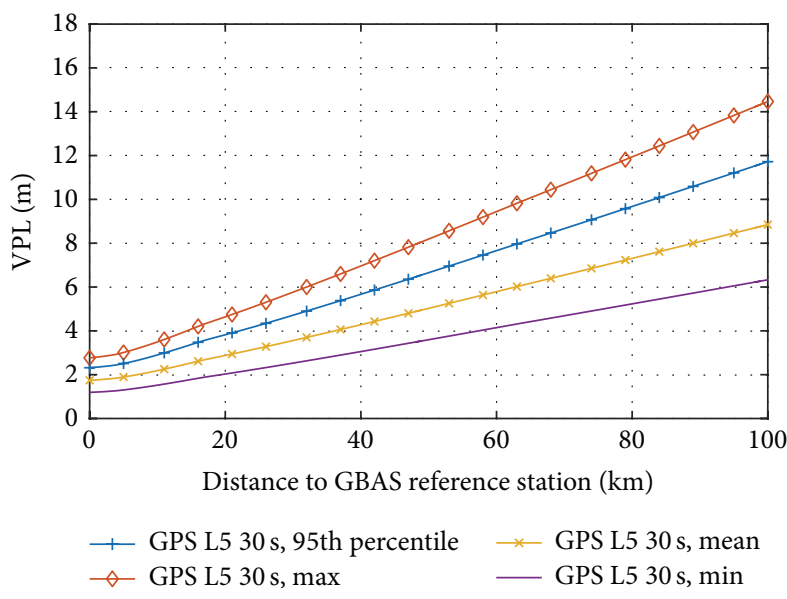

(a) GPS single constellation, L5 $30 \mathrm{~s}$

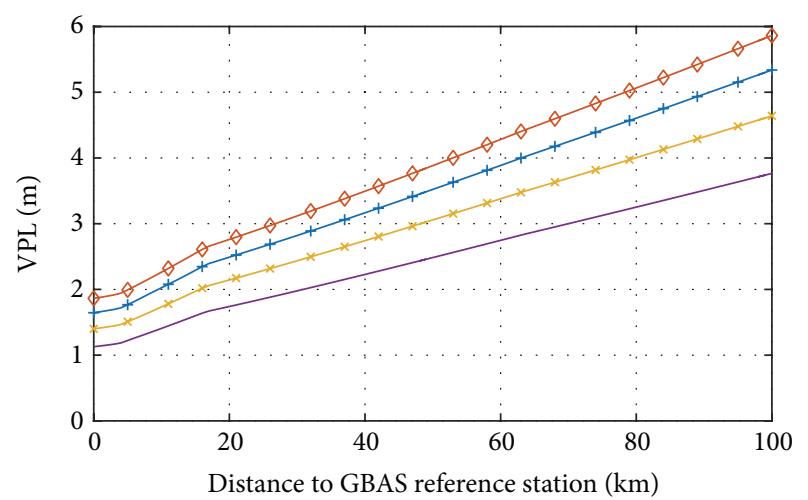

$\begin{array}{ll}\rightarrow \text { DC L1E1 } 100 \text { s, 95th percentile } & \longrightarrow \text { DC L1E1 } 100 \text { s, mean } \\ \neg \text { DC L1E1 } 100 \text { s, max } & \longrightarrow \text { DC L1E1 } 100 \text { s, min }\end{array}$

(b) Dual constellation, L1E1 $100 \mathrm{~s}$

FIGURE 11: Comparison of minimum, maximum, mean, and 95th percentile of H0 VPL over distance during 10 days of simulation.

represents the contribution of $\sigma_{\text {iono }}$. The three plots on the left show different processing modes for a smoothing time constant of 30 seconds while the plots on the right show the corresponding results for 100 seconds. In all cases it is obvious that the longer smoothing time reduces the contributions of $\sigma_{\text {air }}$ and $\sigma_{\text {gnd }}$. As described previously the performance evaluations from ground and airborne measurements showed lowest residual uncertainty for the L5/E5a mode shown in (c) and (d), followed by the L1/E1 mode in (a) and (b) and the largest one in the combined dual frequency mode in (e) and (f). The air and ground contributions are both depending on satellite elevation but not on the baseline between ground station and user. Therefore they remain constant in this plot.

The blue area representing the residual tropospheric uncertainty is the smallest contributor to the protection levels. It is a function of the difference in altitude between the aircraft and the ground station. We simulate an aircraft on a constant $3^{\circ}$ glide path from a level flight at $10000 \mathrm{ft}$. The $\sigma_{\text {tropo }}$ part therefore decreases with smaller distance to the airport.

Finally, the ionospheric contribution greatly varies between the processing modes. For the dual frequency cases (e) and (f) it is constantly zero. Therefore, the protection levels show no significant distance dependency anymore. In the single frequency cases it can be noted that the ionospheric contribution is significantly smaller for the shorter smoothing time constant due to the smoothing time dependent term (18). At short distances the smaller ground and airborne contributions yield smaller protection levels; however at larger distances the ionospheric contribution becomes dominant leading to larger protection levels for the longer smoothing time constant.

There is also a notable difference in how fast the protection levels increase with distance for L1/E1 and L5/E5a. The slope of the green areas is much steeper for the L5/E5a plots and it starts off with a larger initial value at distance $0 \mathrm{~km}$ just due to the greater impact of the ionospheric decorrelation described in (19).

As we already mentioned earlier all simulations in the following section will be carried out using $\sigma_{\text {gnd }}$ models derived from our own measurements which are not using a MLA installation. To give an idea of how the overall system performance in terms of VPL changes with a MLA equipped ground station we include Figure 13. In this figure the same split of VPL contributions as before is performed for GPS Ifree $30 \mathrm{~s}$ and L1 $100 \mathrm{~s}$ processing using the according models from Figure 5(d). It is clearly visible that the contribution of $\sigma_{\text {gnd }}$ alone is significantly dropping. Nevertheless the effect on the overall VPL is less pronounced due to the geometric addition of the different sigma terms, leading to an improvement of about 0.27 (GPS Ifree $30 \mathrm{~s}$ ) to 0.66 (GPS L1 100 a) meters. Almost constant (over distance) improvement in this range can be more or less transferred to the results in Section 5.5 .

5.5. Comparison of Processing Modes. After splitting up the protection level into its individual contributors, we now take a more detailed look at the advantages and performance of the different processing modes. In Figure 14 we show protection level simulations as a function of distance for several potential future GBAS processing modes. Both subfigures include the curve for GAST D for the legacy GBAS CAT-II/III service as baseline.

Figure 14(a) shows the VPL over distance for an assumed smoothing time of 30 seconds for all processing modes. It compares the standard GAST D performance (based on the AAD-B model) and a GAST D curve based on our own new airborne model with three dual constellation curves, namely, single frequency L1/E1, single frequency L5/E5a, and Ifree dual frequency L1/E1 + L5/E5a. The first GAST D curve (green circles) is using the integrity parameters as described in the MOPS.

In this case the bounding is done based on the parameters for 100 -second smoothing and the difference between the 30 -second and 100-second smoothing is accounted for in the $D_{V}$ term as described in (6). As $D_{V}$ is depending on the actual noise encountered at the airborne receiver it is not deterministic and therefore we did not include it in our simulations here. The $D_{V}$-value would be added to the 

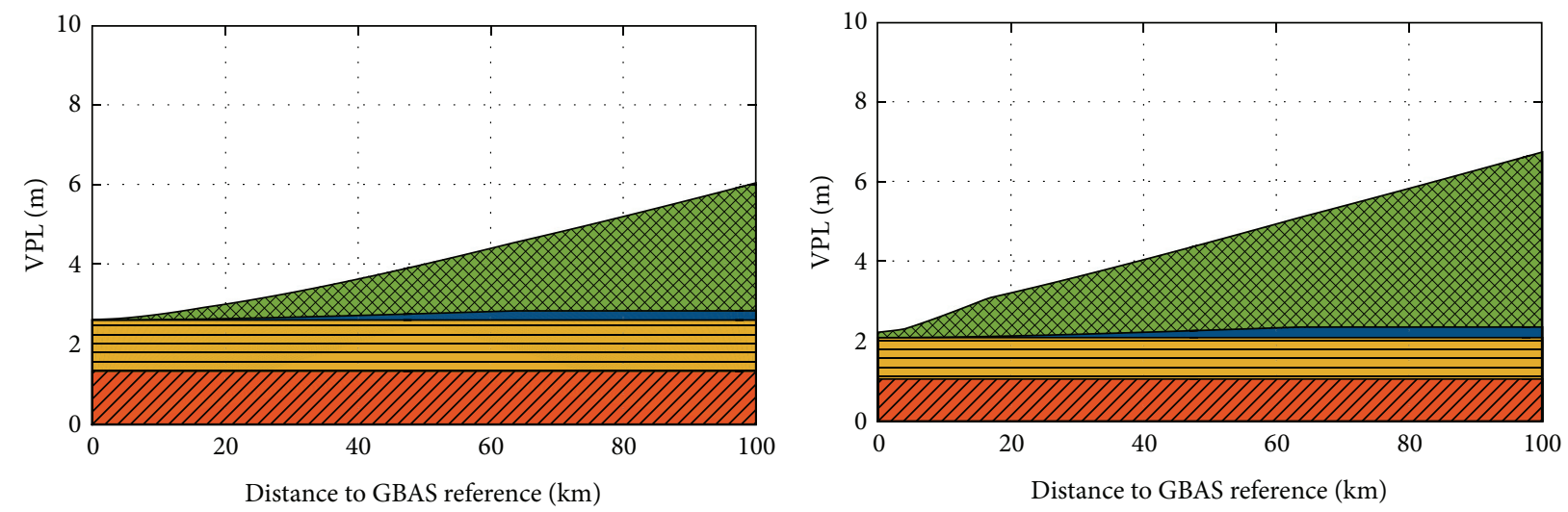

$\begin{array}{ll}\sigma_{\text {gnd }}+\sigma_{\text {air }}+\sigma_{\text {tropo }}+\sigma_{\text {iono }} & \sigma_{\text {gnd }}+\sigma_{\text {air }} \\ \sigma_{\text {gnd }}+\sigma_{\text {air }}+\sigma_{\text {tropo }} & \sigma_{\text {gnd }}\end{array}$

(a) GPS L1 $30 \mathrm{~s}$

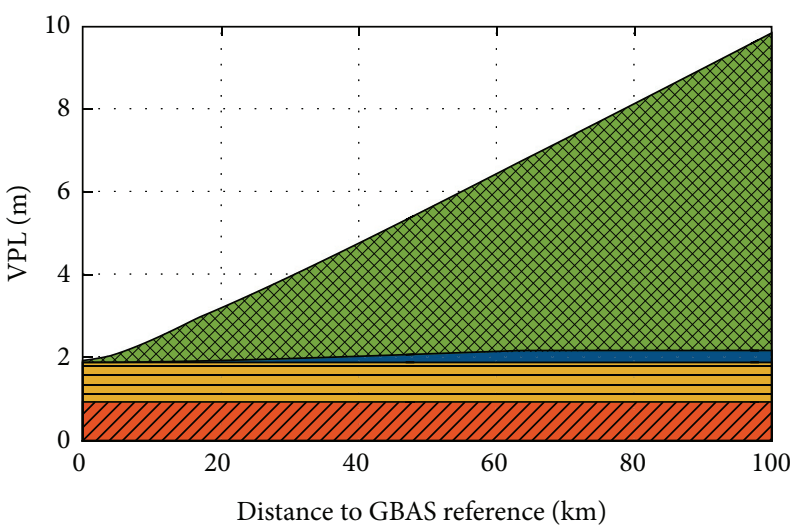

$\begin{array}{ll}\sigma_{\text {gnd }}+\sigma_{\text {air }}+\sigma_{\text {tropo }}+\sigma_{\text {iono }} & \sigma_{\text {gnd }}+\sigma_{\text {air }} \\ \sigma_{\text {gnd }}+\sigma_{\text {air }}+\sigma_{\text {tropo }} & \sigma_{\text {gnd }}\end{array}$

(b) GPS L1 $100 \mathrm{~s}$

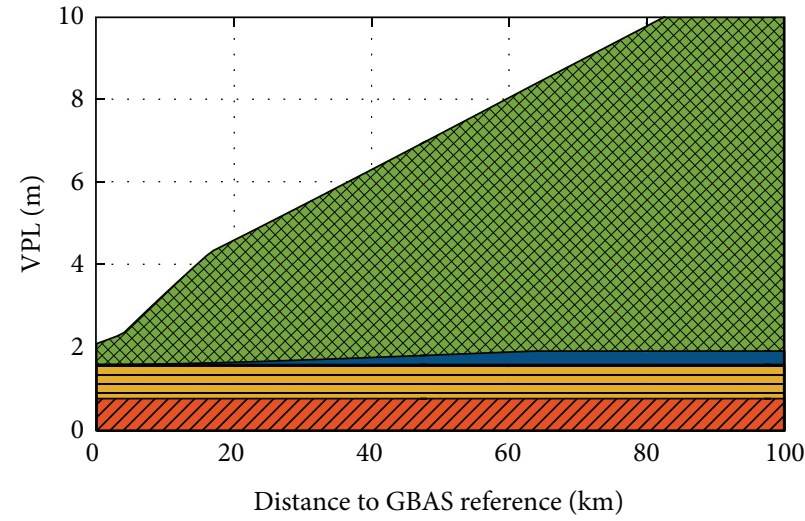

$$
\begin{aligned}
& \sigma_{\text {gnd }}+\sigma_{\text {air }}+\sigma_{\text {tropo }}+\sigma_{\text {iono }} \text { 目 } \sigma_{\text {gnd }}+\sigma_{\text {air }} \\
& \sigma_{\text {gnd }}+\sigma_{\text {air }}+\sigma_{\text {tropo }}
\end{aligned}
$$

(c) GPS L5 $30 \mathrm{~s}$

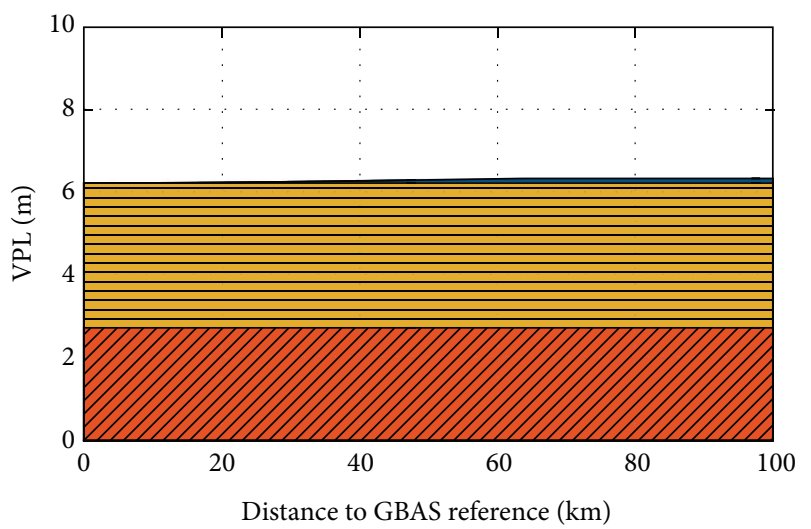

$\sigma_{\text {gnd }}+\sigma_{\text {air }}+\sigma_{\text {tropo }}+\sigma_{\text {iono }}$ 貝 $\sigma_{\text {gnd }}+\sigma_{\text {air }}$
$\sigma_{\text {gnd }}+\sigma_{\text {air }}+\sigma_{\text {tropo }}$

(e) GPS Ifree $30 \mathrm{~s}$

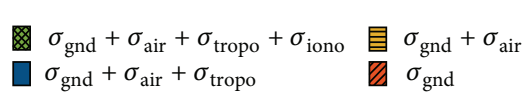

(d) GPS L5 $100 \mathrm{~s}$

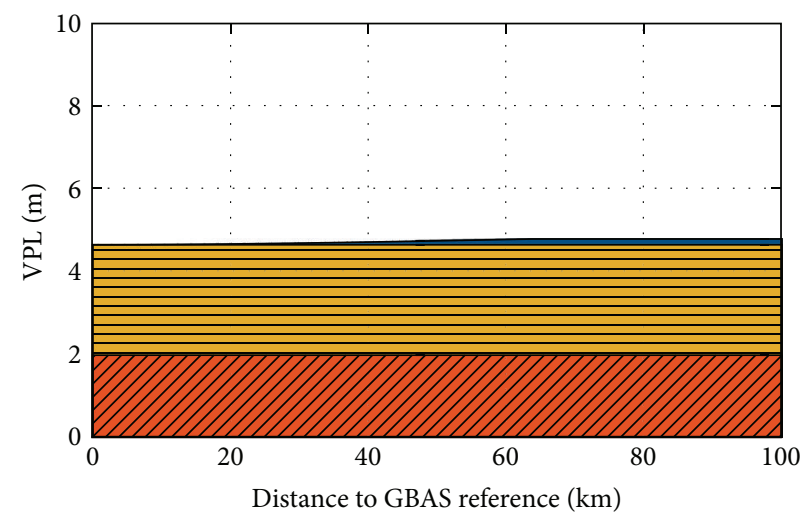

$\sigma_{\text {gnd }}+\sigma_{\text {air }}+\sigma_{\text {tropo }}+\sigma_{\text {iono }}$ 貝
$\sigma_{\text {gnd }}+\sigma_{\text {air }}+\sigma_{\text {tropo }}+\sigma_{\text {air }}$
$\sigma_{\text {gnd }}$

(f) GPS Ifree $100 \mathrm{~s}$

FIGURE 12: H0 protection level contributions of different $\sigma$-terms over distance to the GBAS station. 


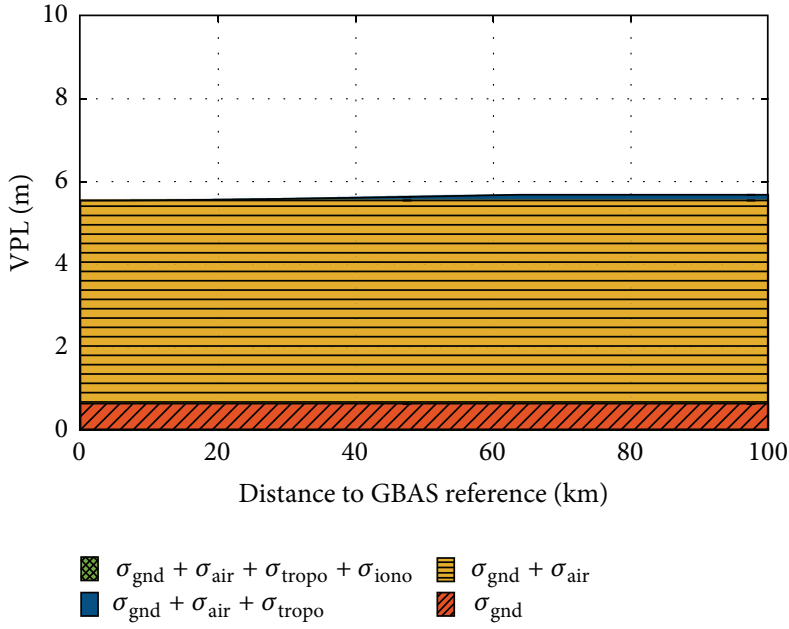

(a) GPS Ifree $30 \mathrm{~s}$

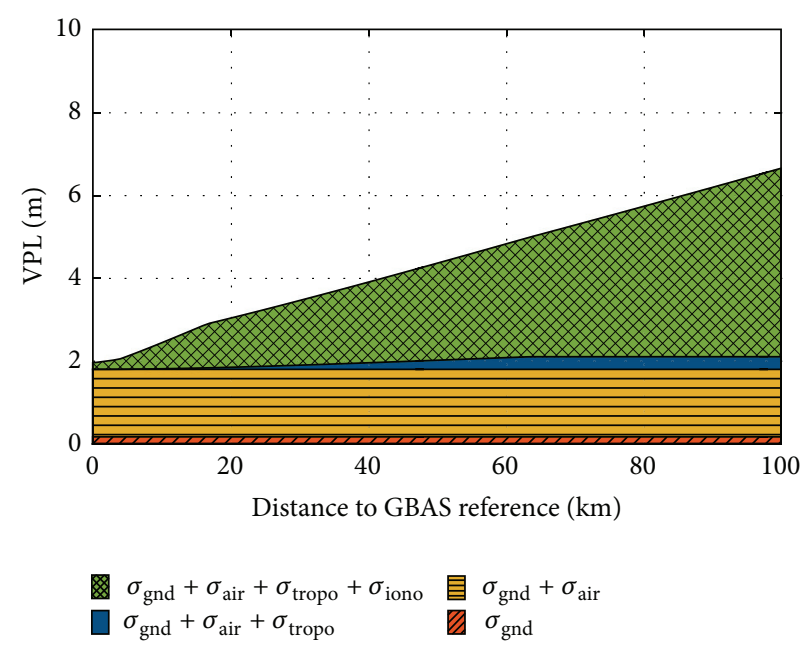

(b) GPS L1 $100 \mathrm{~s}$

FIGURE 13: H0 protection level contributions of different $\sigma$-terms over distance to the GBAS station for a hypothetical MLA antenna.

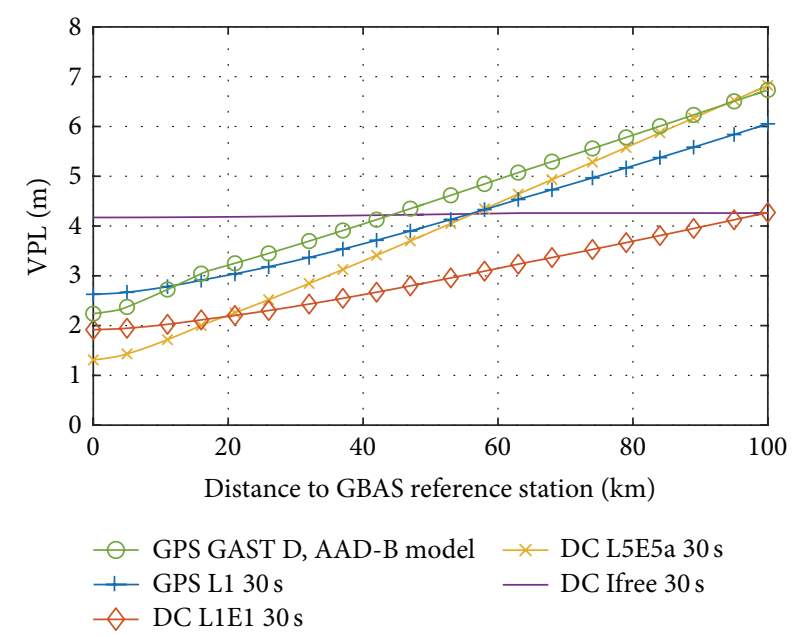

(a) Comparison of GAST D VPLs with different processing modes in 30 s smoothing

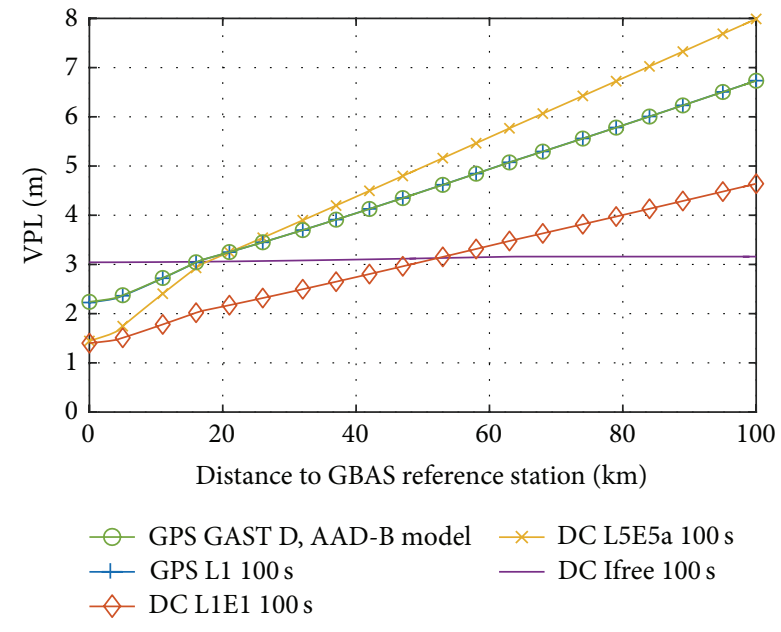

(b) Comparison of GAST D VPLs with different processing modes in 100 s smoothing

FIGURE 14: Comparisons of H0 VPL over distance for sets of different processing modes.

curve shown in the plot and the resulting curve would thus be slightly higher with some variations depending on the changing $D_{V}$. Previous studies found that $D_{V}$ has a standard deviation of around $0.25 \mathrm{~m}$ [6]. The other GAST $\mathrm{D}$ curve shown (blue pluses) represents the protection levels with a $\sigma_{\text {air }}$ model derived from our own measurements on our test aircraft A320 and is based on 30-second smoothed data [15]. Including a second constellation (Galileo in our simulations) and staying with a 30-second smoothing time constant and the L1/E1 signals bring an improvement in terms of performance (red diamonds) compared to the GAST D case. Here, mainly the beneficial effect of a larger constellation and thus a better satellite geometry is visible. A small part of the improvement also results from the somewhat better performance in terms of $\sigma_{\text {air }}$ and $\sigma_{\text {gnd }}$ for the Galileo signals (both again experimentally derived; see Section 4.2).
At short distances between the ground station and a user the best performance is achieved with a dual constellation single frequency L5/E5a mode (yellow crosses). Due to the very low noise and multipath on these signals they have the lowest $\sigma_{\text {air }}$ and $\sigma_{\text {gnd }}$ values which dominate the protection level for short baselines. However, due to the larger ionospheric delay on L5/E5a and thus the inflated $\sigma_{\text {iono }}$ compared to the L1/E1 case the protection levels increase much faster. At just below $20 \mathrm{~km}$ a similar performance as in the dual constellation L1/E1 case is achieved and at distances of about $45-55 \mathrm{~km}$ a similar performance as for the GAST D case is reached. Soon after that even the Ifree protection level is exceeded, making it the largest protection level of all modes in this figure. While all single frequency cases show a significant increase in the protection level over distance, the Ifree protection level remains rather constant. 

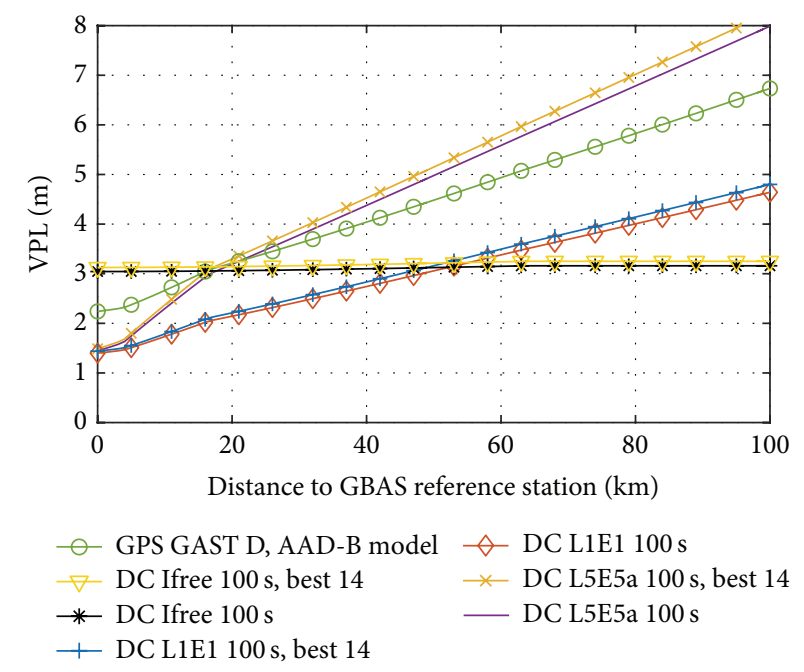

(a) Comparison of GAST D VPLs with different Ifree processing modes

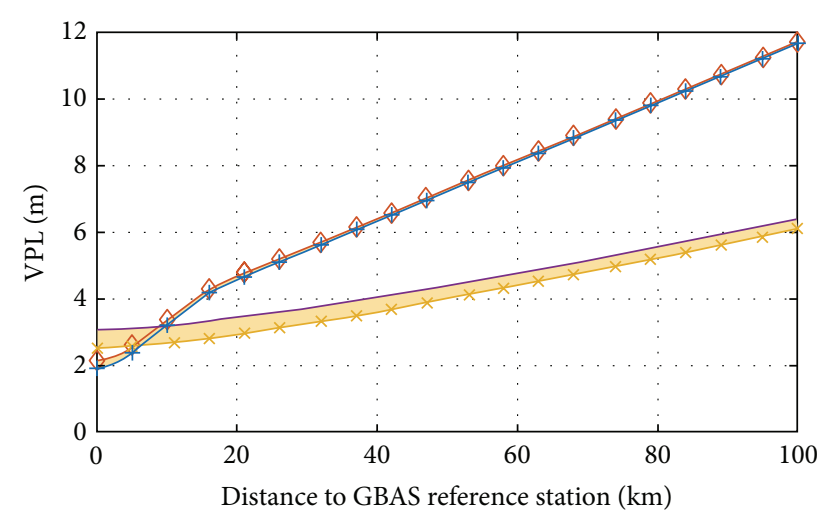

+ Gal E5a 100 s, optimistic $\quad \rightarrow$ Gal E1 30s, optimistic

$\checkmark$ Gal E5a 100 s, conservative — Gal E1 30s, conservative

(b) Galileo E5a (100s) and E1 (30 s) for optimistic as well as conservative $\sigma_{\text {air }}$ model

FIGURE 15: Comparisons of H0 VPL over distance for sets of different processing modes.

This behavior results from the distance dependency of $\sigma_{\text {iono }}$ which is assumed as zero in the dual frequency case. At short distances the Ifree protection is larger due to the dominance of the larger $\sigma_{\text {air }}$ and $\sigma_{\text {gnd }}$ resulting from the combination of two code measurements. However, at larger distances $\sigma_{\text {iono }}$ becomes dominant in the single frequency cases and outweighs the benefits in terms of lower noise and multipath. After about $100 \mathrm{~km}$ distance the Ifree protection level is becoming the best performing one. While this might not be relevant for the approach service this behavior could be exploited when expanding the service volume of GBAS which is currently limited to $42 \mathrm{~km}$.

Figure 14(b) now shows the results for the same processing techniques, however using a smoothing time constant of 100 seconds instead of 30 seconds. In this case the GAST $\mathrm{D}$ model based on the AAD-B model (green circles) and our own experimental model (blue pluses) yield almost identical results. Looking at the four single frequency processing modes GAST D (AAD and experimental model), dual constellation L1/E1 (red diamonds), and dual constellation L5/E5a (yellow crosses) it becomes apparent that they all increase rather fast in the beginning up to a distance of about $16.6 \mathrm{~km}$. This effect results from the simulation of the changing aircraft speed previously explained and illustrated in Figure 7. Again, due to the larger $\sigma_{\text {vig }}$ on L5/E5a the protection level increase in that case is much faster than for the L1/E1 modes. Due to this increase it is also worth noting that the protection levels for the single frequency L5/E5a mode are larger than the L1/E1 protection levels already from the beginning. The larger $\sigma_{\text {vig }}$ multiplied with the longer smoothing time constant increases the $\sigma_{\text {iono }}$ term in the protection levels so much that the noise and multipath benefit on $\sigma_{\text {air }}$ and $\sigma_{\text {gnd }}$ becomes secondary to that increase.

Next, we discuss the effect of using a limited number of satellites instead of the all-in-view solution and the impact of using different models for $\sigma_{\text {air }}$.
In Section 4.1 we explained that it might not always be possible to use all available satellites and showed a way of selecting a good subset of satellites. Figure 15(a) now shows a comparison of the resulting protection levels when using the best 14 satellites out of all available satellites. As for "best" we are referring to the selection according to the heuristic we previously described and discussed. We keep in green circles the GAST D protection level as reference. Additionally we show the protection levels for single frequency L1/E1, single frequency L5/E5a, and the dual frequency Ifree always for the all-in-view and subset of the best 14 satellites for the respective mode. These curves show what was also discussed previously: the selection of a "good" subset has only a very limited impact on the overall geometry. Usually 14 satellites provide a sufficiently good geometry such that the protection level increase remains in the range of few centimeters, especially at short distances between ground station and user.

Figure 15(b) shows the impact different airborne noise and multipath performances have. As we discussed in Section 4.2 about $\sigma_{\text {air }}$ we derived the airborne performance models for the new signals from a limited amount of data and only from one aircraft type. Based on the actual measurements we derived two sets of model curves, one somewhat optimistic and one significantly more conservative. The difference between those curves is shown as shaded area between the purple line and the yellow line with the crosses for the Galileo E1 case and the very small area between the line with the red diamonds markers and the line with the blue pluses for the Galileo E5a case. As previously explained, we found it sufficient to derive one model for Galileo E5a and GPS L5 since the signals have the same modulation and chipping rate. For GPS L1 the model differs from the Galileo E1 due to the different modulations that are used. The difference between the conservative and the optimistic model is larger for the 30second smoothed signals than for the 100 -second smoothed signals. Thus, of course the impact of choosing one of the two 


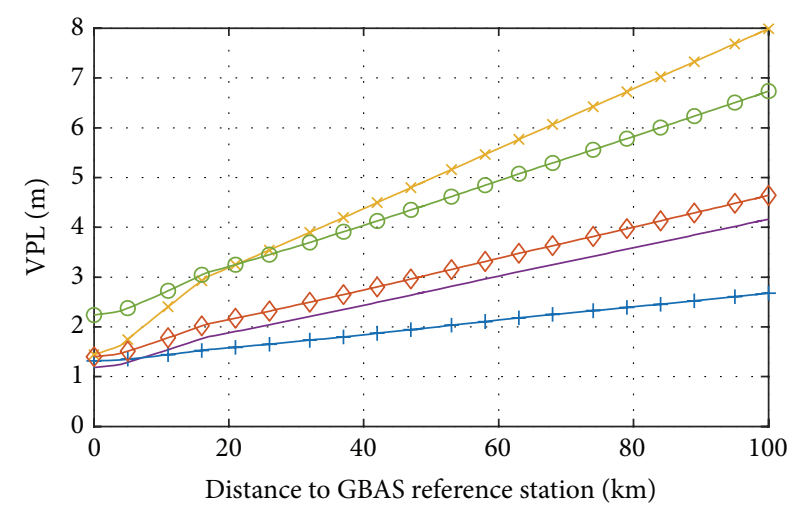

$\begin{array}{ll}\odot \text { GPS GAST D, ADD-B model } & - \text { DC L1E1 } 100 \mathrm{~s}, \sigma_{\text {vig }}=2 e-6 \\ \smile \text { DC L1E1 } 100 \mathrm{~s}, \sigma_{\text {vig }}=4 e-6 & - \text { DC L5E5a } 100 \mathrm{~s}, \sigma_{\text {vig }}=2 e-6 \\ \star \text { DC L5E5a } 100 \mathrm{~s}, \sigma_{\text {vig }}=4 e-6 & \end{array}$

(a) VPL over distance for two different $\sigma_{\text {vig }}$ assumptions

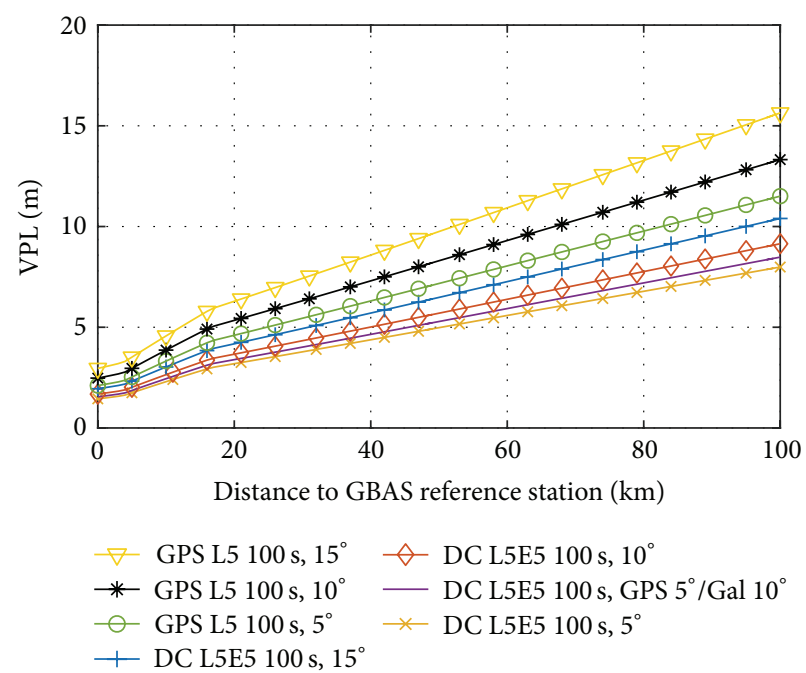

(b) VPLs over distance for different elevation masks and for DC as well as GPS

FIGURE 16: Comparisons of H0 VPL over distance for changing elevation mask and $\sigma_{\text {vig }}$.

models is larger for the 30-second smoothing case, while the difference in the 100-second case is almost negligible. As in the previous plots in Figure 14 the change of the speed up to $16.6 \mathrm{~km}$ is much more visible in the 100 -second smoothing case due the larger effect on $\sigma_{\text {iono }}$.

Finally, in Figure 16 we investigate the impact of using different ionospheric models and the impact of applying different elevation masks. As shown in (18) one central parameter for the calculation of the ionospheric uncertainty is the parameter $\sigma_{\mathrm{vig}}$. It describes the nominal decorrelation of the ionospheric delay over distance between the user and the GBAS ground station. In the United States data analysis led to suggesting a nominal decorrelation of $4 \mathrm{~mm} / \mathrm{km}$ [16], while for Germany a value of $2.07 \mathrm{~mm} / \mathrm{km}$ was found to sufficiently bound the observations [2]. Figure 16(a) shows the effect on the protection levels when using those two values and again the curve with the green circles as GAST D baseline for comparison. The two lines for 100-second smoothing with $\sigma_{\text {vig }}$ of $4 \mathrm{~mm} / \mathrm{km}$ with the red diamonds and yellow crosses are the same as in Figure 14(a). It is interesting to note that when changing $\sigma_{\text {vig }}$ to $2 \mathrm{~mm} / \mathrm{km}$ for distances of up to $7 \mathrm{~km}$ (and thus where typically the aircraft would touch down) the L5/E5a combination yields the best results and not the L1/E1 mode as with $4 \mathrm{~mm} / \mathrm{km}$. Generally, the smaller $\sigma_{\text {vig }}$ is very beneficial at larger distances to the airport where the size of the protection levels is significantly decreased as the $\sigma_{\text {iono }}{ }^{-}$ term becomes more and more dominant.

Finally, the effect of applying elevation masking is shown in Figure 16(b).

In Section 4.1 we already discussed how a raised elevation mask affects the DOP values in a dual constellation case. However, in the GBAS context it may also be beneficial to exclude low elevation satellites as the expected error variance increases with decreasing elevation. Furthermore, most multipath and noise (reflected in $\sigma_{\text {air }}$ and $\sigma_{\text {gnd }}$ ) affect those low elevation satellites. Excluding them might also ease the requirements on the antennas used in the ground and airborne systems in the future.

A different aspect is the loss of satellites when the aircraft is maneuvering. It was observed that low elevation satellites are often lost in turns. Even after reacquisition it takes some time until the smoothing filters have converged and the satellite can be reincluded into the position solution. Particularly, when the benefits of GBAS in terms of flexible paths and curved approaches are going to be fully exploited in the future, this factor will have an impact on the overall performance. Using satellites less likely to be affected (i.e., higher elevation satellites) reduces the impact on the navigation performance.

For current GBAS the elevation mask is set to $5^{\circ}$ according to [18]. However, in the Galileo interface control document [19], a $10^{\circ}$ elevation mask for Galileo is recommended. As this limit might be relaxed in the future we consider elevation masks for GPS and Galileo of $5^{\circ}, 10^{\circ}$, and $15^{\circ}$.

It can be clearly seen that no matter which of these types of elevation masking is chosen the four dual constellation cases always yield better results than the GPS standalone mode with $5^{\circ}$. Applying a $5^{\circ}$ mask to GPS and a $10^{\circ}$ mask to Galileo (purple line without markers) decreases the protection levels slightly compared to the $5^{\circ}$ elevation mask for all constellations scenario (orange crosses). Raising the elevation mask to $10^{\circ}$ for all constellations still yields good results as the number of satellites available for navigation is still large. However, of course applying higher elevation masks to GPS only decreases the number of satellites usable and thus degrades geometry leading to larger protection levels.

\section{Conclusions}

In this study we investigated the performance of different processing modes for GBAS, including single and dual frequency and single and dual constellation modes. This was 


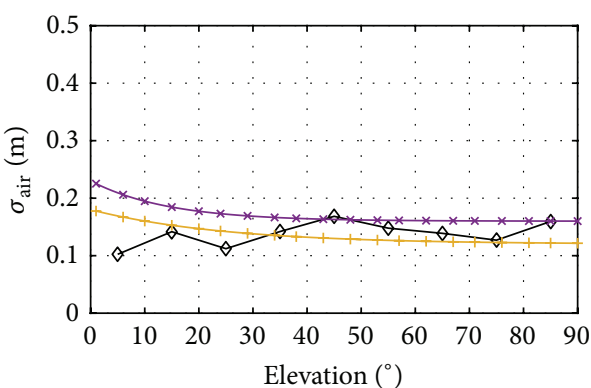

$\vdash \sigma_{\text {air }}-$ optimistic model $\neg-\sigma_{\text {Gal El, 100s }}$ $* \sigma_{\text {air }}-$ conservative model

(a) Galileo E1 $100 \mathrm{~s}$

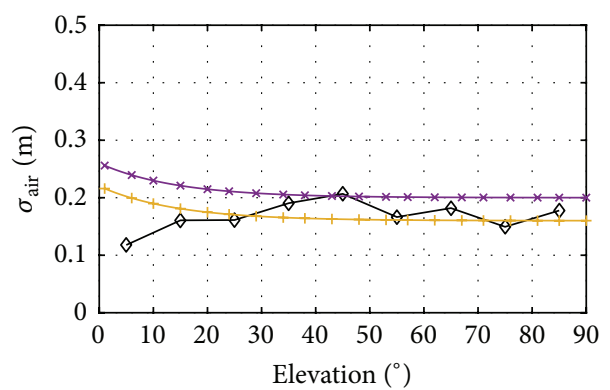

$\vdash \sigma_{\text {air }}-$ optimistic model $\neg-\sigma_{\mathrm{GPS} \text { L5/Gal E5,30s }}$ $* \sigma_{\text {air }}$-conservative model

(c) GPS L5/Galileo E5a $30 \mathrm{~s}$

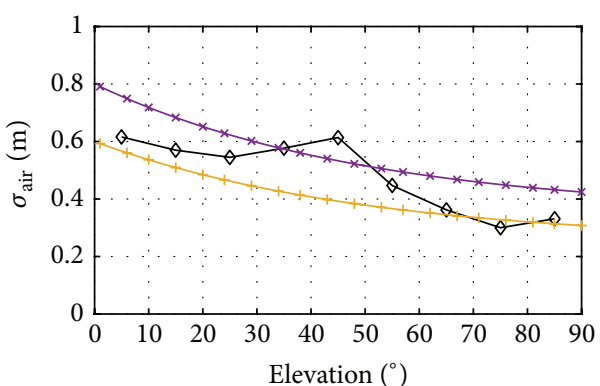

$+\sigma_{\text {air }}-$ optimistic model $\neg-\sigma_{\mathrm{GPS} \text { Ifree, } 100 \mathrm{~s}}$ $\star \sigma_{\text {air }}-$ conservative model

(e) GPS Ifree $100 \mathrm{~s}$

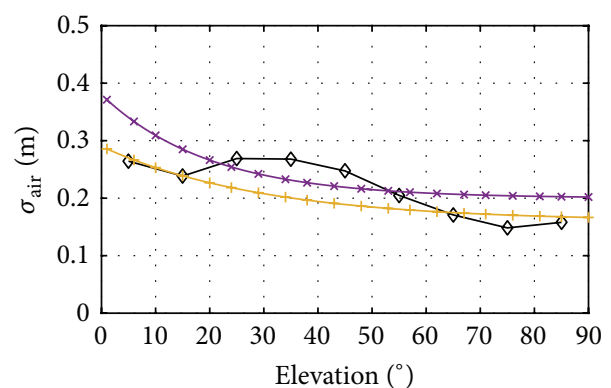

$+\sigma_{\text {air }}-$ optimistic model $\smile \sigma_{\mathrm{GPS} L 1,100 \mathrm{~s}}$ $\star \sigma_{\text {air }}-$ conservative model

(b) GPS L1 $100 \mathrm{~s}$

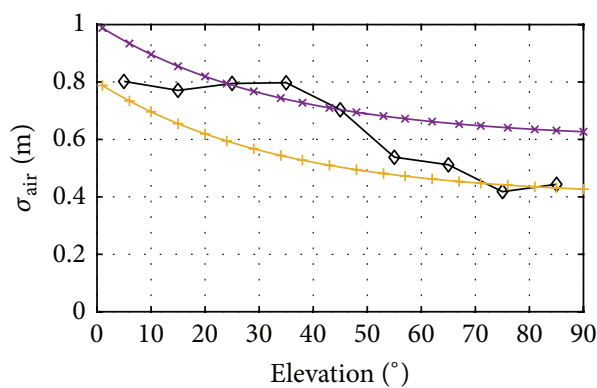

$\dashv \sigma_{\text {air }}-$ optimistic model $\frown \sigma_{\mathrm{GPS} \text { Ifree, } 30 \mathrm{~s}}$ $* \sigma_{\text {air }}-$ conservative model

(d) GPS Ifree $30 \mathrm{~s}$

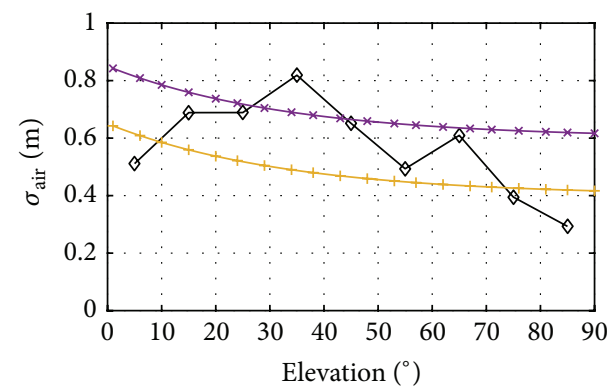

$+\sigma_{\text {air }}-$ optimistic model $\neg-\sigma_{\text {Gal Ifree, 30 s }}$ $\star \sigma_{\text {air }}-$ conservative model

(f) Galileo Ifree $30 \mathrm{~s}$

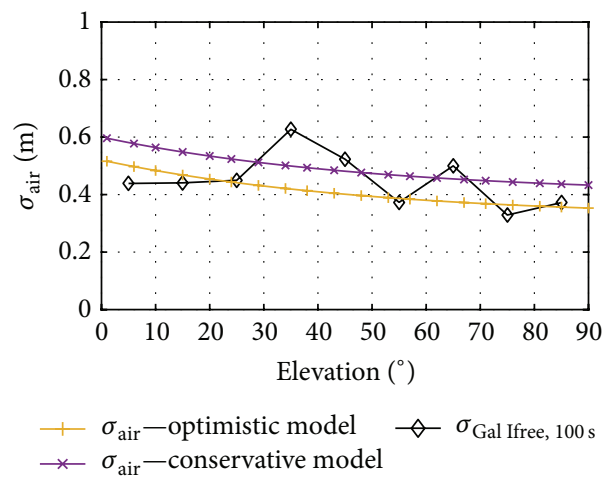

(g) Galileo Ifree $100 \mathrm{~s}$

FIGURE 17: $\sigma_{\text {gnd }}$ curves for different processing schemes. 
achieved combining results from previous studies with new analysis on airborne measurements and the development of first preliminary airborne models. The work contributes to trade-off studies in terms of the selection of future GBAS processing schemes. As the results show, the performance improves when using a second constellation, resulting in smaller protection levels with significantly less variations over time. This is the case for using two full constellations and when only selecting a good subset (e.g., the best 14 satellites) of all available satellites. Furthermore, it was found that an increased elevation mask to $10^{\circ}$ does usually not affect the resulting protection level by much, especially at short distances of up to $20 \mathrm{~km}$ from the airport.

The definition of an optimal (in the sense of smallest protection levels) processing scheme for future dual constellation and dual frequency GBAS is a complex issue and depends on many parameters. Single frequency processing modes all have to account for the ionospheric decorrelation with distance and for the additional divergence introduced by the smoothing filter. The Ifree dual frequency processing eliminates the ionospheric issue. On the other hand, the noise and multipath performance is significantly better in the single frequency modes than for the dual frequency case. Thus, the protection levels close to the GBAS station tend to be small for the single frequency cases but increase with distance and speed of the aircraft. The Ifree dual frequency mode has a rather constant performance with respect to distance between airport and user. Ifree protection levels are larger when the aircraft is close to the airport but the single frequency protection levels increase with distance and eventually exceed the dual frequency protection levels. The use of single frequency techniques is therefore beneficial at short distances, for example, during the final approach, but the Ifree processing yields smaller protection levels at larger distances. This is relevant especially for extending the current GBAS service volume to distances larger than $43 \mathrm{~km}$. For operations at larger distances the VPL will, however, not be the decisive quantity anymore. Typically, the ephemeris protection level then becomes dominant. Furthermore, it may be decided that at those distances only lateral guidance will be provided by GBAS and vertical guidance is based on barometric altimetry.

From the single frequency modes the susceptibility to ionospheric decorrelation is larger on L5/E5a than on L1/E1. Therefore, the ionospheric uncertainty increases faster with distance and speed of the aircraft. However, the noise and multipath performance is better on L5/E5a than on L1/E1. Depending on the assumed nominal ionospheric decorrelation, the smoothing time constant and the speed profile of the aircraft of either mode may yield smaller protection levels and better performance at short baselines. However, with increasing distance and speed, the larger ionospheric uncertainty lets the L5/E5a protection levels grow faster than the L1/E1 ones. For making a final decision on the best architecture of primary and backup processing modes of a future GBAS it is therefore necessary to decide on standard model parameters, such that a final conclusion can be reached.

\section{Competing Interests}

The authors declare that there is no conflict of interests regarding the publication of this paper.

\section{References}

[1] S. Pullen, Y. S. Park, and P. Enge, "Impact and mitigation of ionospheric anomalies on ground-based augmentation of GNSS," Radio Science, vol. 44, no. 1, 2009.

[2] C. Mayer, B. Belabbas, N. Jakowski, M. Meurer, and W. Dunkel, "Ionosphere threat space model assessment for GBAS," in Proceedings of the 22nd International Technical Meeting of the Satellite Division of the Institute of Navigation (ION GNSS '09), pp. 217-225, Savannah, Ga, USA, September 2009.

[3] M. Kim, Y. Choi, H.-S. Jun, and J. Lee, "GBAS ionospheric threat model assessment for category I operation in the Korean region," GPS Solutions, vol. 19, no. 3, pp. 443-456, 2015.

[4] J. Lee, M. Yoon, S. Pullen et al., "Preliminary results from ionospheric threat model development to support GBAS operations in the Brazilian region," in Proceedings of the 28th International Technical Meeting of the Satellite Division of the Institute of Navigation (ION GNSS+ '15), Tampa Convention Center, Tampa, Fla, USA, September 2015.

[5] N. Hlubek, J. Berdermann, V. Wilken et al., "Scintillations of the GPS, GLONASS, and Galileo signals at equatorial latitude," Journal of Space Weather and Space Climate, vol. 4, article A22, 2014.

[6] T. Dautermann, M. Felux, and A. Grosch, "Approach service type D evaluation of the DLR GBAS testbed," GPS Solutions, vol. 16, no. 3, pp. 375-387, 2012.

[7] RTCA DO-253C, "Minimum operational performance standards for GPS local area augmentation system airborne equipment," Tech. Rep. 253C, Radio Technical Commission for Aeronautics, 2008.

[8] S. Thoelert, M. Vergara, M. Sgammini et al., "Characterization of nominal signal distortions and impact on receiver performance for GPS (IIF) L5 and Galileo (IOV) E1 /E5a signals," in Proceedings of the 27th International Technical Meeting of the Satellite Division of the Institute of Navigation (ION GNSS+ '14), Tampa, Fla, USA, September 2014.

[9] M. Felux, M.-S. Circiu, B. Belabbas et al., "Concept for a dual frequency dual constellation GBAS," in Proceedings of the 28th International Technical Meeting of the Satellite Division of the Institute of Navigation (ION GNSS+ '15), Tampa Convention Center, Tampa, Fla, USA, September 2015.

[10] M. Felux, M.-S. Circiu, D. Gerbeth, M. Caamano, and M. Stanisak, "Ionospheric monitoring in a dual frequency GBAS," in Proceedings of the IEEE Aerospace Conference 2016, IEEE Aerospace Conference, Big Sky, Mont, USA, March 2016.

[11] ICAO NSP, "GBAS CAT II/III development baseline SARPs," Tech. Rep., International Civil Aviation Organization, 2010.

[12] D. Gerbeth, M. Felux, M.-S. Circiu, and M. Caamano, "Optimized selection of satellite subsets for a multi-constellation GBAS," in Proceedings of the International Technical Meeting of the Institute of Navigation, pp. 360-367, Monterey, Calif, USA, January 2016.

[13] M.-S. Circiu, M. Felux, S. Thölert et al., "Evaluation of GPS L5 and Galileo E1 and E5a performance for future multi frequency and multi constellation GBAS," in Proceedings of the International Technical Meeting of The Institute of Navigation, pp. 374-382, Dana Point, Calif, USA, January 2015. 
[14] G. A. McGraw, T. Murphy, M. Brenner et al., "Development of the LAAS accuracy models," in Proceedings of the 13th International Technical Meeting of the Satellite Division of the Institute of Navigation (ION GPS '00), pp. 1212-1223, Salt Lake City, Utah, USA, September 2000.

[15] M.-S. Circiu, M. Felux, B. Belabbas et al., "Evaluation of GPS L5, Galileo E1 and Galileo E5a performance in flight trials for multi frequency multi constellation GBAS," in Proceedings of the ION GNSS+ 2015, Tampa, Fla, USA, September 2015, http://elib.dlr.de/98863.

[16] J. Lee, S. Pullen, S. Datta-Barua, and P. Enge, "Assessment of ionosphere spatial decorrelation for global positioning systembased aircraft landing systems," Journal of Aircraft, vol. 44, no. 5, pp. 1662-1669, 2007.

[17] T. Murphy, M. Harris, and L. Azoulai, Proposed Modification for GAST D Anomalous Ionosphere Gradient Requirements, ICAO NSP/CSG Flimsy, 2015.

[18] Eurocae, Minimum Operational Performance Specification for Global Navigation Satellite Ground Based Augmentation System Ground Equipment to Support Category I Operations, Eurocae, 2013.

[19] Galileo Open Service Signal-in-Space Interface Control Document 1.2, September 2015, http://www.gsc-europa .eu/gnss-markets/segments-applications/os-sis-icd. 


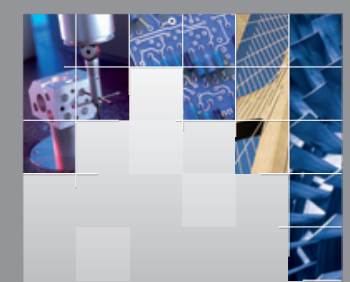

\section{Enfincering}
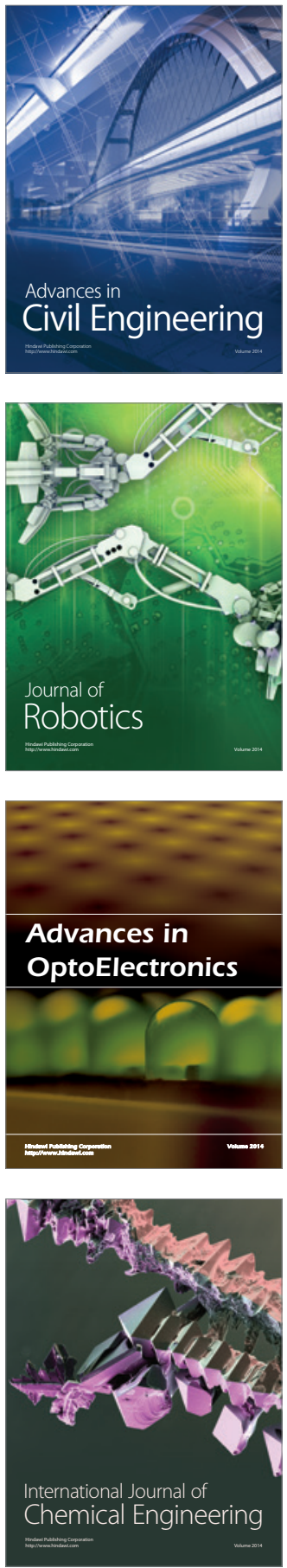

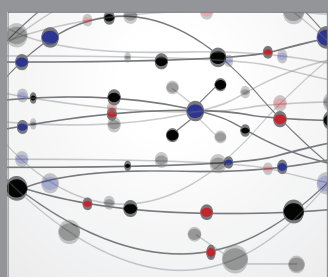

The Scientific World Journal

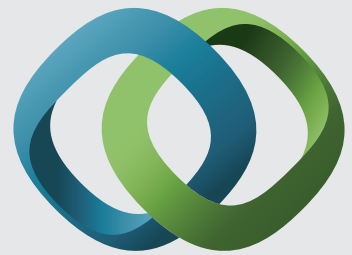

\section{Hindawi}

Submit your manuscripts at

http://www.hindawi.com
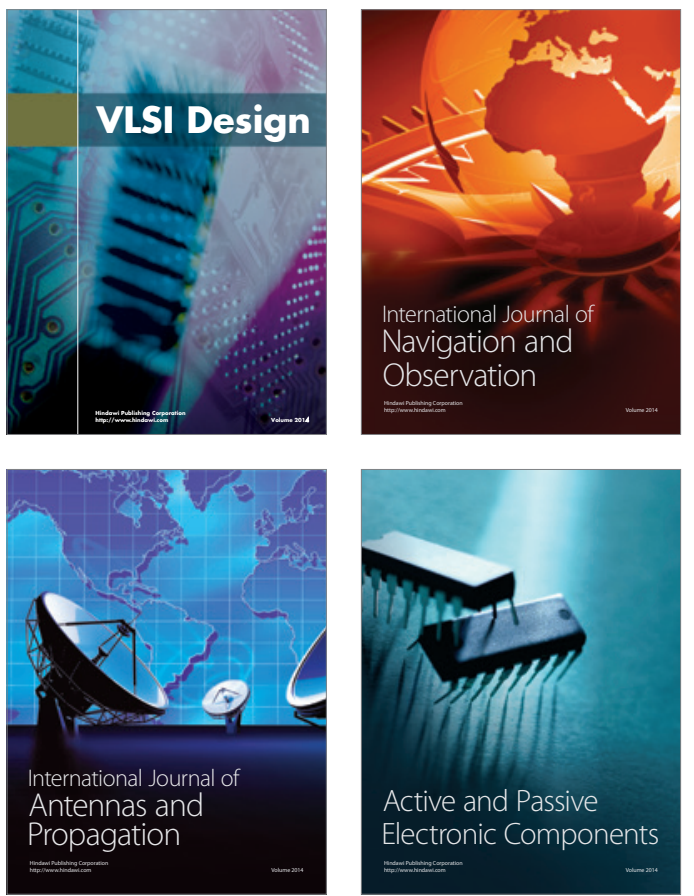
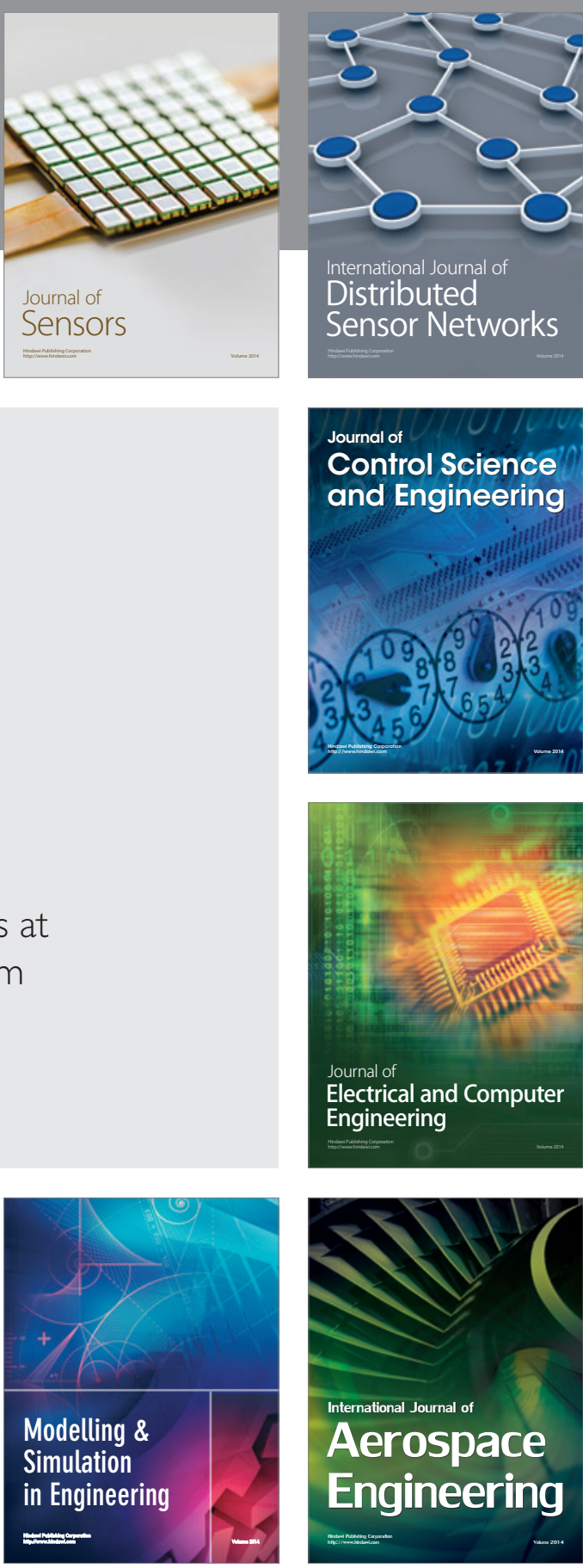

International Journal of

Distributed

Sensor Networks

Journal of

Control Science

and Engineering
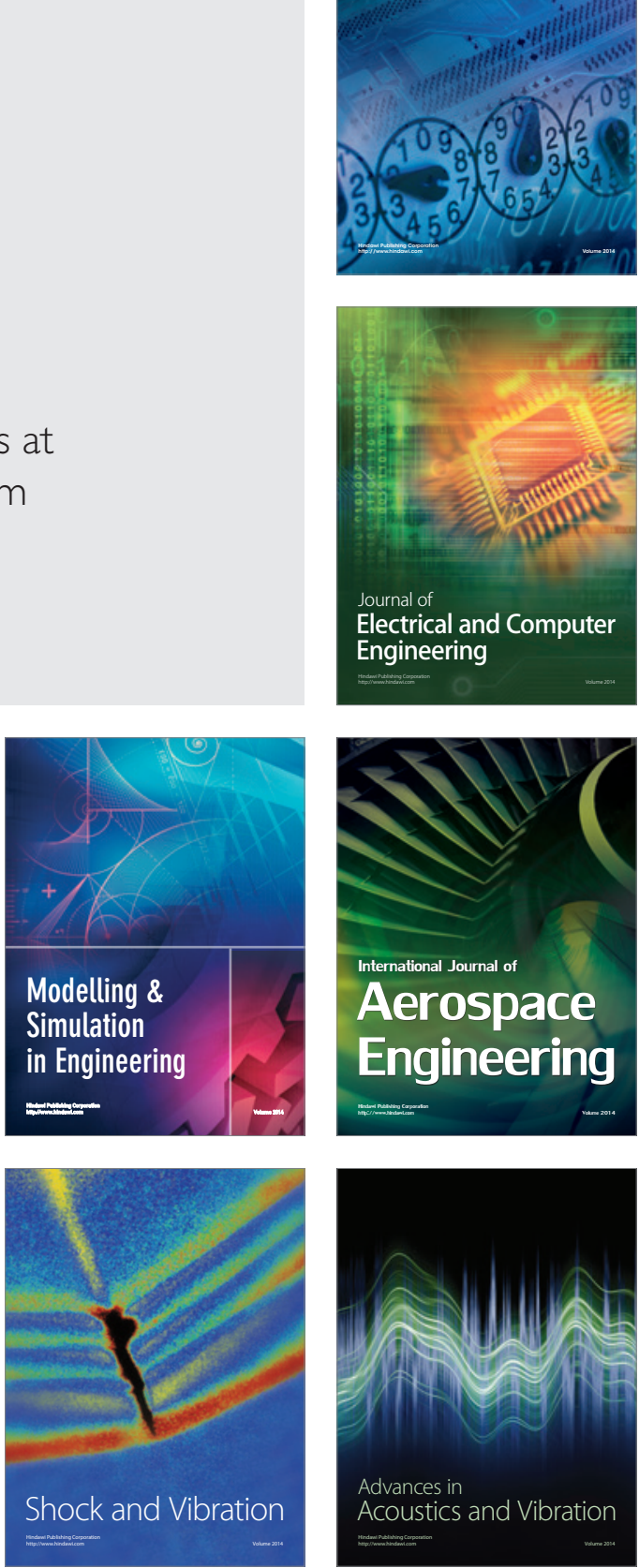